TRANSACTIONS OF THE

AMERICAN MATHEMATICAL SOCIETY

Volume 350, Number 11, November 1998, Pages 4341-4365

S $0002-9947(98) 01775-9$

\title{
SECOND-ORDER CONDITIONS IN EXTREMAL PROBLEMS. THE ABNORMAL POINTS
}

\author{
A. V. ARUTYUNOV
}

\begin{abstract}
In this paper we study a minimization problem with constraints and obtain first- and second-order necessary conditions for a minimum. Those conditions - as opposed to the known ones - are also informative in the abnormal case. We have introduced the class of 2-normal constraints and shown that for them the "gap" between the sufficient and the necessary conditions is as minimal as possible. It is proved that a 2-normal mapping is generic.
\end{abstract}

\section{INTRODUCTION}

1. In this paper we study the following minimization problem with constraints:

$$
\left\{\begin{array}{l}
f(x) \rightarrow \min , x \in X \\
F(x) \in C
\end{array}\right.
$$

Here $X$ is a vector space, $C$ a closed convex cone with a finite number of faces (defined by a finite number of equalities and inequalities) in the $k$-dimensional arithmetic space $Y=R^{k}$, and

$$
f: X \rightarrow R^{1}, F: X \rightarrow Y=R^{k}
$$

smooth mappings (in the sense explained below). Note that (1.1) includes problems of mathematical programming with both equality-and inequality-type constraints. The principal burdensome assumption in the problem (1.1) is that of the finite dimensionality of $Y$, which is the image of the mapping $F$ that represents constraints.

2. Let $x_{0}$ be the solution of the problem (1.1). Then the Lagrange multipliers rule holds for that point $[3,6]$. That means that there exists a collection of Lagrange multipliers $\lambda=\left(\lambda^{0}, y\right)$ such that

$$
\frac{\partial \mathcal{L}}{\partial x}\left(x_{0}, \lambda\right)=0, \lambda^{0} \geq 0, y \in N_{C}\left(F\left(x_{0}\right)\right), \lambda \neq 0 .
$$

Here

$$
\mathcal{L}(x, \lambda)=\lambda^{0} f(x)+\langle y, F(x)\rangle
$$

is the Lagrange function and $N_{C}(z)$ is the normal cone to the set $C$ at a point $z$.

Received by the editors January 25, 1995 and, in revised form, September 20, 1995.

1991 Mathematics Subject Classification. Primary 49B27.

Key words and phrases. Abnormal point, Lagrange multipliers, second-order necessary conditions, index of quadratic form, 2-normal mapping.

This work was supported by Russian Fund of Fundamental Researches, project N 96-01-00800 and partially by Grant of Goskomvuz of Russia, project N 95-0-1.9-48.

(C)1998 American Mathematical Society 
As is known (see, for example, [1]), if one wants to obtain necessary extremal conditions for finite- or infinite-dimensional problems, the most unpleasant difficulties may appear in the case when the point $x_{0}$ is abnormal ${ }^{1}$. That means

$$
\exists y_{0} \in N_{C}\left(F\left(x_{0}\right)\right) \cap\left(-N_{C}\left(F\left(x_{0}\right)\right)\right): y_{0} \neq 0, F^{\prime}\left(x_{0}\right)^{*} y_{0}=0 .
$$

Here $N_{C}(y) \cap\left(-N_{C}(y)\right)$ is the largest subspace contained in the cone $N_{C}(y)$.

The fact is that abnormal points are singular for the manifold $\{x \in X: F(x) \in$ $C\}$ and the conditions (1.2) trivially hold for an abnormal point, that is, with Lagrange multipliers $\lambda=\left(\lambda^{0}, y\right)$ such that $\lambda^{0}=0$ and $y=y_{0}$, where $y_{0}$ is any vector that satisfies the conditions (1.3). Thus (1.2) holding at an abnormal point for an arbitrary objective functional $f$ is only a trivial consequence of the abnormality condition and does not provide us with any information. Moreover, the classical second-order necessary condition, which requires the second derivative of the Lagrange function to be nonnegative on the cone

$$
\left\{h \in X:\left\langle f^{\prime}\left(x_{0}\right), h\right\rangle \leq 0, F^{\prime}\left(x_{0}\right) h \in T_{C}\left(F\left(x_{0}\right)\right)\right\}
$$

(where $T_{C}(y)=N_{C}^{0}(y)$ is the tangent cone to the set $C$ at a point $y$ ), generally does not hold for an abnormal point. This may be substantiated by the following example:

$$
\begin{gathered}
f(x)=-\left(x_{1}^{2}+x_{2}^{2}\right) \rightarrow \min ; x=\left(x_{1}, x_{2}\right) \in R^{2} ; \\
F(x)=\left(F_{1}(x), F_{2}(x)\right)=0, F_{1}(x)=x_{1} x_{2}, \\
F_{2}(x)=x_{1}^{2}-x_{2}^{2} ; x_{0}=0 .
\end{gathered}
$$

Let us cite a specialist in second-order conditions on the normality condition: "It is natural to ask what means we have at our disposal if the normality hypothesis is not satisfied. The corresponding generalization of the theory seems difficult or even impossible" [2].

3. In this paper $(\S 3)$ we have obtained second-order necessary conditions that remain informative for abnormal points. If a point under consideration for an extremum is normal (i.e. there is no vector $y_{0}$ that would satisfy (1.3)), then the conditions turn out to be well known. Our derivation of the necessary conditions is based on an estimate from below of the upper topological limit of a sequence of subspaces. The estimate is calculated in $\S 2$.

Further, in $\S 4$ we introduce the notion of 2-normal mapping at a point (with respect to the cone $C$ ). We prove that if the mapping $F$ is 2-normal at a point, then the second-order necessary conditions become sufficient by means of an arbitrarily small quadratic perturbation of the mappings $f$ and $F$. Hence the "gap" between the second-order sufficient and second-order necessary conditions is as minimal as possible at the points where the constraint is 2-normal. The value of this "gap" is a very important characteristic of extremal conditions. The fifth section deals with the notion of 2-normality. We obtain effectively verifiable sufficient conditions of 2-normality at a point. We prove that if $X=R^{n}$ and $n \gg k$, then the set of the mappings $F$ that are 2-normal at every point with respect to any cone $C$ is everywhere dense in the space $C_{s}^{3}\left(R^{n}, R^{k}\right)$ with the Whitney topology.

\footnotetext{
${ }^{1}$ If $C=0$, then the Lyusternic condition is said to be violated at the point $x_{0}$.
} 


\section{Notation and assumptions. Auxiliary tools}

1. Let angular brackets $\langle$,$\rangle denote the scalar product and the action of a linear$ functional. Furthemore, if $\varphi$ is a set, then $|\varphi|$ is the number of its elements. $|x|$ denotes the modulus of a vector $x$. Lin is the linear hull and conv the convex hull of a set. $M^{\perp}$ is the orthogonal complement or annihilator of a subspace $M$. The set

$$
N_{C}(y)=\{\xi \in Y:\langle\xi, z-y\rangle \leq 0 \forall z \in C\}
$$

is the normal cone to a convex set $C$ at a point $y \in C$, and $T_{C}(y)=N_{C}^{0}(y)$ the tangent cone to $C$ at a point $y$, where ${ }^{0}$ is the cone polar. By ${ }^{*}$ we denote the conjugate operator and the algebraically dual space. If $\left\{\Pi_{n}\right\}$ is a sequence of topological space subsets, then $L s\left\{\Pi_{n}\right\}$ denotes its upper topological limit, which, as we know, consists of all possible limit points of the sequences $\left\{x_{n}\right\}$ with $x_{n} \in$ $\Pi_{n} \forall n$. If $A$ is a quadratic form or a quadratic mapping, we set $A[x]^{2}=\langle A x, x\rangle$. Let $q$ be a quadratic form and $Z$ a subspace of $X$. Then $\operatorname{ind}_{Z} q$ is the index of the form $q$ on $Z$ (i.e. the maximal of the dimensions of the subspaces $N \subseteq Z$ on which the form $q$ is negative-definite).

Let us introduce the so-called finite topology in the vector space $X$. In this topology we regard as open only a set whose intersection with any finite-dimensional subspace $M$ is open in the linear (the only separable) topology of $M$. Denote the finite topology by $\tau$. It is the strongest topology among those which turn $X$ into a topological vector space. On the other hand, the minimum local with respect to the finite topology is the weakest in comparison with any other kind of minimum accepted for consideration.

Fix a point $x_{0} \in X$. The functions $f$ and $F=\left(F_{1}, \ldots, F_{k}\right)$ are assumed to be twice continuously differentiable in a neighborhood of the point $x_{0}$ with respect to the finite topology $\tau$. This means that for an arbitrary finite-dimensional linear subspace $M$ the restrictions of $f$ and $F$ to $M$ are twice continuously differentiable in a neighborhood of $x_{0}$ (the neighborhood depends on $M$ ). Thus there exist a linear functional $a \in X^{*}$, a linear operator $A: X \rightarrow Y$, a quadratic form $q: X \rightarrow R^{1}$, a quadratic mapping $Q: X \rightarrow Y$ and, in addition, mappings $\alpha_{0}: X \rightarrow R^{1}$, $\alpha: X \rightarrow R^{k}$ such that

$$
\begin{gathered}
f(x)=f\left(x_{0}\right)+\left\langle a, x-x_{0}\right\rangle+\frac{1}{2} q\left(x-x_{0}\right)+\alpha_{0}\left(x-x_{0}\right), \\
F(x)=F\left(x_{0}\right)+A\left(x-x_{0}\right)+\frac{1}{2} Q\left(x-x_{0}\right)+\alpha\left(x-x_{0}\right) \forall x \in X,
\end{gathered}
$$

and for an arbitrary finite-dimensional subspace $M$

$$
\frac{\left\|\alpha\left(x-x_{0}\right)\right\|_{M}}{\left\|x-x_{0}\right\|_{M}^{2}} \rightarrow 0, x \rightarrow x_{0}, x \in M
$$

where \|\|$_{M}$ is the finite-dimensional norm on $M$. The last condition is also true for $\alpha_{0}$. The mappings $a$ and $q$ will be denoted by $f^{\prime}\left(x_{0}\right)$ (or $\frac{\partial f}{\partial x}\left(x_{0}\right)$ ) and $f^{\prime \prime}\left(x_{0}\right)$ (or $\frac{\partial^{2} f}{\partial x^{2}}\left(x_{0}\right)$ ) and called the first and second derivatives of $f$. We use analogous notation in the case of the mapping $F$.

2. Let us obtain an estimate from below for the upper topological limit of a sequence of subspaces. Let $l_{\infty}^{k}$ be the Banach space of bounded sequences of $k$ dimensional vectors $\left\{x^{n}\right\}_{n=1}^{\infty}, x^{n} \in R^{k} \forall n$, with the usual sup-norm. We begin by constructing a continuous linear operator that acts from $l_{\infty}^{k}$ into $R^{k}$ and possesses all the properties that we need for further investigations. A. Ya. Dubovitskii was 
the first to prove the existence of such an operator for $k=1$. He communicated that fact to the author in a personal discussion.

Let $N$ be the set of positive integers and $\varphi_{0}$ an arbitrary infinite subset of $N$. Then there exists a nontrivial ultrafilter $\Phi$ in $N$ that contains $\varphi_{0}$ [4] (the nontriviality of $\Phi$ means that the intersection of all its elements is empty).

To prove $|\varphi|=\infty \forall \varphi \in \Phi$, let us assume the contrary. Then among subsets $\varphi \in \Phi$ with a finite number of elements we can choose $\tilde{\varphi}$ with the smallest cardinal. Let us consider an arbitrary $\varphi \in \Phi$. We therefore have $\tilde{\varphi} \subseteq \varphi$, because if $\tilde{\varphi}$ does not belong to $\varphi$, then $\tilde{\varphi} \cap \varphi \in \Phi,|\tilde{\varphi} \cap \varphi|<|\tilde{\varphi}|$, which contradicts the choice of $\tilde{\varphi}$. Thus $\tilde{\varphi} \subseteq \varphi \forall \varphi \in \Phi$ and, consequently, the intersection of all the elements $\varphi \in \Phi$ is not empty (because the nonempty set $\tilde{\varphi}$ belongs to it). This contradiction to the nontriviality of $\Phi$ proves that $|\varphi|=\infty \forall \varphi \in \Phi$.

The ultrafilter $\Phi$ generates a premeasure (i.e. a finitely additive function) $d$ on $2^{N}$ by the formula

$$
d(A)= \begin{cases}1, & \text { if } A \in \Phi, \\ 0, & \text { if } A \notin \Phi ; A \in 2^{N} .\end{cases}
$$

We will show that $d$ is finitely additive. Indeed, let $A, B \in 2^{N}$. One can prove that

$$
d(A \cup B)+d(A \cap B)=d(A)+d(B) .
$$

If $A \in \Phi, B \in \Phi$, then $A \cup B \in \Phi, A \cap B \in \Phi$, and (2.2) is obvious. If $A \notin \Phi$, $B \notin \Phi$, then, from the properties of the ultrafilter, we get $A \cup B \notin \Phi \Rightarrow A \cap B \notin \Phi$ and (2.2) is obvious. If $A \in \Phi, B \notin \Phi$, then $A \cup B \in \Phi ; A \cap B \notin \Phi$ and (2.2) obviously holds again. Hence $d$ is a premeasure. Let us observe that $d\left(\varphi_{0}\right)=1$, by construction.

Lemma 2.1 (A. Ya. Dubovitskii). Let $\varphi$ be an arbitrary infinite subset of $N$. Then there exists a continuous linear operator $\mathcal{D}_{\varphi}^{k}: l_{\infty}^{k} \rightarrow R^{k}$ such that if $\mathcal{D}_{\varphi}^{k} x=0$, then zero is a limit point of a sequence $\left\{x^{n}\right\}, n \in \varphi$.

Proof. Let us consider a nontrivial ultrafilter $\Phi$ that contains $\varphi$, and define the premeasure $d$ according to the formula (2.1). Further, we define a linear continuous operator $\mathcal{D}_{\varphi}^{k}: l_{\infty}^{k} \rightarrow R^{k}$ with the help of the premeasure following the scheme employed to build the integral on the base of a finitely additive measure on $L_{\infty}$. Namely, let $x \in l_{\infty}^{k}$ be a simple sequence, that is, there exists a partition of $N$ into mutually disjoint subsets $A_{1}, \ldots, A_{r}$ such that $x^{n}=a_{i} \forall n \in A_{i}, i=1, \ldots, r$ where $a_{i}$ are given vectors from $R^{k}$. Set $\mathcal{D}_{\varphi}^{k} x=\sum_{n=1}^{r} d\left(A_{i}\right) a_{i}$. By the standard procedure it can be verified that the value of $\mathcal{D}_{\varphi}^{k}$ on a simple sequence does not depend on the way of partitioning $N$ into $A_{i}$. Since the simple sequences subspace is everywhere dense in $l_{\infty}^{k}$, the operator $\mathcal{D}_{\varphi}^{k}$ can be extended over all $l_{\infty}^{k}$ by continuity.

We assert that the operator $\mathcal{D}_{\varphi}^{k}$ is the required one. Indeed, if $\mathcal{D}_{\varphi}^{k} x=0$, then for an arbitrary $\varepsilon>0$ there exist $x_{\varepsilon} \in l_{\infty}^{k}$ (which depends on $\varepsilon$ ), $r \in$ $N, a_{i} \in R^{k}$ and mutually disjoint subsets $A_{i} \subseteq N, i=1, \ldots, r$, such that $N=\bigcup_{i=1}^{r} A_{i} ;\left\|x-x_{\varepsilon}\right\|_{l_{\infty}^{k}} \leq \varepsilon ; x_{\varepsilon}^{n}=a_{i} \forall n \in A_{i}$. Then $\mathcal{D}_{\varphi}^{k} x_{\varepsilon}=\sum_{i=1}^{r} a_{i} d\left(A_{i}\right)$. From the properties of the ultrafilter it follows [4] that there is only one set among the $A_{i}$ (for example $A_{i^{\prime}}$ ) that belongs to $\Phi$. That fact and (2.1) yield 


$$
\begin{aligned}
\mathcal{D}_{\varphi}^{k} x_{\varepsilon}=a_{i^{\prime}} \Rightarrow\left|a_{i^{\prime}}\right| & =\left|\mathcal{D}_{\varphi}^{k} x_{\varepsilon}\right|=\left|\mathcal{D}_{\varphi}^{k} x_{\varepsilon}-\mathcal{D}_{\varphi}^{k} x\right| \leq\left\|\mathcal{D}_{\varphi}^{k}\right\| \varepsilon=\varepsilon \\
& \Rightarrow\left|x^{n}\right| \leq 2 \varepsilon \forall n \in A_{i^{\prime}} .
\end{aligned}
$$

But by construction, $\varphi \in \Phi \Rightarrow \varphi \cap A_{i^{\prime}} \in \Phi$ and, as proved earlier, $\varphi \cap A_{i^{\prime}}$ contains an infinite number of indices $n$ for which $\left|x^{n}\right| \leq 2 \varepsilon$. Hence, the arbitrariness of $\varepsilon>0$ allows us to conclude that the statement is true.

Remark. In Lemma 2.1 we have not proved the existence of an operator but of the whole family of different Dubovitskii operators $\mathcal{D}_{\varphi}^{k}$, because every infinite subset $\varphi \subseteq N$ generates a nontrivial (in fact, not unique) ultrafilter employed in (2.1) to define a premeasure, which is then used to build an operator. However, if the sequence $x=x^{n}$ converges, then the value of any Dubovitskii operator at $x$ is equal to $\lim _{i \rightarrow \infty} x^{i}$. Let us denote the operator $\mathcal{D}_{N}^{k}$ by $\mathcal{D}^{k}$.

3. Let $X$ be a Banach space, $\left\{A_{n}\right\}_{n=1}^{\infty}$ a sequence of continuous linear operators that act from $X$ into $Y=R^{k}$. We assume that the sequence $\left\{A_{n}\right\}$ converges in norm to a linear operator $A: X \rightarrow Y$. Set $M=L s\left\{\operatorname{Ker} A_{n}\right\}$. It is clear that $M$ is closed, nonempty $(0 \in M)$ and $M \subseteq \operatorname{Ker} A$. If the operator $A$ is surjective, then by the theorem proved below $M=\operatorname{Ker} A$. If $A$ is not surjective, then $M \neq \operatorname{Ker} A$ in general, and the set $M$ may not even be convex.

Theorem 2.1. In the space $X$ there exists a closed subspace $\Pi$ such that codim $\Pi \leq$ $k ; \Pi \subseteq L s \operatorname{Ker} A_{n}$ and $\Pi \subseteq \operatorname{Ker} A$.

Proof. Let $P_{1}: Y \rightarrow \operatorname{Im} A, P_{2}: Y \rightarrow(\operatorname{Im} A)^{\perp}$ denote the corresponding orthogonal projection operators. Set

$$
\begin{gathered}
r=\operatorname{codim}(\operatorname{Im} A) ; L_{n}=P_{1} \circ A_{n} ; L=P_{1} \circ A ; \\
x_{i, n}^{*}=y_{i}^{*} \circ P_{2} \circ A_{n}, i=1, \ldots, r .
\end{gathered}
$$

Here $\left\{y_{i}^{*}\right\}$ is a basis of the space dual to $(\operatorname{Im} A)^{\perp}$. By the continuity of the projection operators, the linear operators $L_{n}$ and the functionals $x_{i, n}^{*}$ are continuous. Moreover, $L_{n} \rightarrow L, n \rightarrow \infty$ and $L$ is surjective. We turn the topologically dual spaces $X^{\prime}$ and $Y^{\prime}$ into Banach spaces in the usual way, using the same symbol \| \| to denote the norms in them and identifying $Y$ with $Y^{\prime}\left(Y=R^{k}\right)$.

Using the definition of the number $r$, we assume without loss of generality that for each number $n$ the restrictions of linear functionals $x_{i, n}^{*}, i=1, \ldots, r$, to the subspace Ker $L_{n}$ are linearly independent (otherwise we could consider a suitable subsequence). Thus, taking into consideration the finite dimensionality of $Y$, we easily find that for each number $i=1, \ldots, r, n=1,2, \ldots$, the solution of the problem

$$
\left\|x_{i, n}^{*}+\sum_{j=1}^{i-1} \alpha_{j} x_{j, n}^{*}+L_{n}^{*} y\right\| \rightarrow \inf , \alpha_{j} \in R^{1}, y \in Y,
$$

is positive and reachable. Hence there exist $y_{i, n} \in Y$ and numbers $\alpha_{j, i, n}$ such that

$$
\begin{gathered}
\bar{x}_{i, n}^{*}=x_{i, n}^{*}+\sum_{j=1}^{i-1} \alpha_{j, i, n} x_{j, n}^{*}+L_{n}^{*} y_{i, n} ; \\
\bar{x}_{i, n}^{*} \neq 0 ; \quad\left\|\bar{x}_{i, n}^{*}+\sum_{j=1}^{i-1} \alpha_{j} x_{j, n}^{*}+L_{n}^{*} y\right\| \geq \frac{1}{2}\left\|\bar{x}_{i, n}^{*}\right\| \forall\left(\alpha_{j}, y\right) .
\end{gathered}
$$


It follows from the last inequality that

$$
\left\|\tilde{x}_{i, n}^{*}+\sum_{j=1}^{i-1} \alpha_{j} \tilde{x}_{j, n}^{*}+L_{n}^{*} y\right\| \geq \frac{1}{2} \forall\left(\alpha_{j}, y\right),
$$

where $\tilde{x}_{i, n}^{*}=\bar{x}_{i, n}^{*}\left\|\bar{x}_{i, n}^{*}\right\|^{-1}$, for, by construction,

$$
\tilde{x}_{i, n}^{*} \in \operatorname{Lin}\left(x_{1, n}^{*}, \ldots, x_{i, n}^{*}, \operatorname{Im} L_{n}^{*}\right) .
$$

We also see that

$$
\bigcap_{i=1}^{r} \operatorname{Ker} x_{i, n}^{*} \cap \operatorname{Ker} L_{n}=\bigcap_{i=1}^{r} \operatorname{Ker} \tilde{x}_{i, n}^{*} \cap \operatorname{Ker} L_{n} .
$$

Let us define a linear operator $\Phi: X \rightarrow l_{\infty}^{r}$ by setting

$$
\Phi x=\left\{\left(\begin{array}{c}
\left\langle\tilde{x}_{1, n}^{*}, x\right\rangle \\
\vdots \\
\left\langle\tilde{x}_{r, n}^{*}, x\right\rangle
\end{array}\right)\right\}_{n=1}^{\infty} .
$$

Let $\mathcal{D}^{r}$ be a Dubovitskii operator whose existence was proved in Lemma 2.1. We set

$$
\tilde{\Pi}=\left\{x \in X: \mathcal{D}^{r} \circ \Phi x=0\right\}, \Pi=\tilde{\Pi} \cap \operatorname{Ker} A,
$$

and show that the subspace $\Pi$ is the required one.

Indeed, $\Pi \subseteq \operatorname{Ker} A$ and

$$
\operatorname{codim} \Pi \leq \operatorname{codim} \tilde{\Pi}+\operatorname{codim}(\operatorname{Ker} A) \leq r+\operatorname{dim}(\operatorname{Im} A)=k .
$$

Therefore it is enough to prove that if $x_{0} \in \Pi$, then $x_{0}$ is a limit point for a sequence of points $\left\{x_{n}\right\}$ with $x_{n} \in \operatorname{Ker} A_{n}$.

Indeed, let $\mathcal{D}^{r} \circ \Phi x_{0}=0$. Then, with the aid of Lemma 2.1 and considering a suitable subsequence, we have

$$
\left\langle\tilde{x}_{i, n}^{*}, x_{0}\right\rangle \rightarrow 0, n \rightarrow \infty, i=1, \ldots, r .
$$

For each $n$ we examine the problem:

$$
J_{n}(x)=\left\|x-x_{0}\right\|^{2} \rightarrow \inf ; L_{n} x=0,\left\langle\tilde{x}_{i, n}^{*}, x\right\rangle=0, i=1, \ldots, r .
$$

Let $J_{n}$ be the infinum. We choose $\tilde{x}_{n} \in X$ that complies with all the constraints of that problem and for which

$$
J_{n}\left(\tilde{x}_{n}\right) \leq J_{n}+\frac{1}{n^{2}}
$$

Then according to the $\varepsilon$-sub-differential theorem (see, for example, [21]) we conclude that for large numbers $n$ there exist $x_{n} \in X, h_{n}^{*} \in X^{*}$ and Lagrange multipliers $\lambda_{i, n} \in R^{1}, i=1, \ldots, r$, and $y_{n} \in(\operatorname{Im} A)^{*}$ such that

$$
\sum_{i=1}^{r} \lambda_{i, n} \tilde{x}_{i, n}^{*}+L_{n}^{*} y_{n}+h_{n}^{*} \in \partial \kappa\left(x_{n}-x_{0}\right)
$$

and

$$
\left\|x_{n}-\tilde{x}_{n}\right\| \leq 1 / n,\left\|h_{n}^{*}\right\| \leq 1 / n, L_{n} x_{n}=0,\left\langle\tilde{x}_{i, n}^{*}, x_{n}\right\rangle=0, i=1, \ldots, r .
$$

Here $\partial \kappa$ is the sub-differential of the convex function $\kappa(x)=\|x\|^{2}$. From [3],

$$
\left\|x^{*}\right\|=2\|x\| ;\left|\left\langle x^{*}, x\right\rangle\right|=2\|x\|^{2} \forall x^{*} \in \partial \kappa(x) .
$$


It follows from (2.7) and the first formula of (2.8) that

$$
2\left\|x_{n}-x_{0}\right\| \geq\left\|\sum_{i=1}^{r} \lambda_{i, n} \tilde{x}_{i, n}^{*}+L_{n}^{*} y_{n}\right\|-1 / n,
$$

which along with (2.4) implies

$$
\left|\lambda_{i, n}\right| \leq \operatorname{const}\left(\left\|x_{n}-x_{0}\right\|+\frac{1}{n}\right), i=1, \ldots, r .
$$

According to the Banach open mapping theorem and the surjectivity of $L$, we have

$$
\left\|L^{*} y\right\| \geq \text { const }\|y\| \forall y \in \operatorname{Im} A \Rightarrow\left\|L_{n}^{*} y\right\| \geq \text { const }\|y\| \forall y \in \operatorname{Im} A
$$

for sufficiently large $n$. Consequently, by (2.7) and the first formula of (2.8),

$$
\left\|y_{n}\right\| \leq \operatorname{const}\left(\left\|x_{n}-x_{0}\right\|+\frac{1}{n}\right) \text {. }
$$

Applying the left part of (2.7) to the vector $\left(x_{n}-x_{0}\right)$ and using (2.8), we obtain

$$
2\left\|x_{n}-x_{0}\right\|^{2} \leq \operatorname{const}\left(\left\|x_{n}-x_{0}\right\| \sum_{i=1}^{r}\left|\left\langle\tilde{x}_{i, n}^{*}, x_{0}\right\rangle\right|+\frac{1}{n}\right),
$$

for $\left\langle\tilde{x}_{i, n}^{*}, x\right\rangle=0, i=1, \ldots, r$. From this and (2.6) it follows that $x_{n} \rightarrow x_{0}$ as $n \rightarrow \infty$. Since

$$
\left\langle\tilde{x}_{i, n}^{*}, x_{n}\right\rangle=0, i=1, \ldots, r
$$

and $L_{n} x_{n}=0$, by $(2.5)$,

$$
\left\langle x_{i, n}^{*}, x_{n}\right\rangle=0, i=1, \ldots, r \Rightarrow x_{n} \in \operatorname{Ker} A_{n} .
$$

The theorem is proved.

Theorem 2.2. Let $\left\{\Pi_{n}\right\}$ be a sequence of closed subspaces from $X$ with codim $\Pi_{n} \leq$ $k \forall n$. Then there exists a closed subspace $\Pi \subseteq X$ such that codim $\Pi \leq k$ and $\Pi \subseteq L s\left\{\Pi_{n}\right\}$.

Proof. Let us choose continuous linear operators $\tilde{A}_{n}: X \rightarrow Y$ such that Ker $\tilde{A}_{n}=$ $\Pi_{n} \forall n$. Set $A_{n}=\tilde{A}_{n}\left(n\left\|\tilde{A}_{n}\right\|\right)^{-1}$. It is clear that $A_{n} \rightarrow 0$ as $n \rightarrow \infty$. We prove the statement by applying Theorem 2.1 to $\left\{A_{n}\right\}$.

Remark. In [5] the author obtained estimates from below for $L s\left\{x: A_{n} x \in \mathcal{K}\right\}$ where $\mathcal{K}$ is a cone with a finite number of faces in a Banach space.

\section{SECOND-ORDER NECESSARY CONDITIONS}

1. We return to the problem (1.1). Throughout this section we assume that the point $x_{0}$ is a local minimum with respect to the finite topology $\tau$, while $f$ and $F$ are continuously differentiable functions in a neighborhood of $x_{0}$ with respect to the finite topology.

Consider the Lagrange function

$$
\mathcal{L}(x, \lambda)=\lambda^{0} f(x)+\langle y, F(x)\rangle ; \lambda=\left(\lambda^{0}, y\right), \lambda^{0} \in R^{1}, y \in Y .
$$

By the Lagrange multipliers rule (which gives first-order necessary conditions), for the local minimum point $x_{0}$ there exist Lagrange multipliers $\lambda$ such that

$$
\frac{\partial \mathcal{L}}{\partial x}\left(x_{0}, \lambda\right)=0, \lambda^{0} \geq 0, y \in N_{C}\left(F\left(x_{0}\right)\right), \lambda \neq 0 .
$$


The set of all the Lagrange multipliers that correspond to the point $x_{0}$ forms a nonempty (nonclosed) cone $\Lambda=\Lambda\left(x_{0}\right)$ such that $\Lambda \cup\{0\}$ is convex.

Let us consider a subset of $\Lambda$. $\lambda$ belongs to that subset if, and only if, there exists a subspace $\Pi \subseteq X$ such that

$$
\operatorname{codim} \Pi \leq k ; \Pi \subseteq \operatorname{Ker} F^{\prime}\left(x_{0}\right) ; \frac{\partial^{2} \mathcal{L}}{\partial x^{2}}\left(x_{0}, \lambda\right)[x]^{2} \geq 0 \forall x \in \Pi .
$$

We shall denote that subset of Lagrange multipliers by $\Lambda_{a}=\Lambda_{a}\left(x_{0}\right)$. It is clear that $\Lambda_{a} \subseteq \Lambda$ and $\Lambda_{a}$ is a cone (but in a lot of interesting cases the cone $\Lambda_{a} \cup\{0\}$ is not convex).

Notice that the existence of a space $\Pi$ that satisfies (3.2) is equivalent to the condition

$$
\operatorname{ind}_{Z} \frac{\partial^{2} \mathcal{L}}{\partial x^{2}}\left(x_{0}, \lambda\right) \leq \operatorname{codim}\left(\operatorname{Im} F^{\prime}\left(x_{0}\right)\right), Z=\operatorname{Ker} F^{\prime}\left(x_{0}\right) .
$$

We consider the cone

$$
\mathcal{K}=\mathcal{K}\left(x_{0}\right)=\left\{h \in X:\left\langle f^{\prime}\left(x_{0}\right), h\right\rangle \leq 0, F^{\prime}\left(x_{0}\right) h \in T_{C}\left(F\left(x_{0}\right)\right)\right\} .
$$

Theorem 3.1. The set $\Lambda_{a}\left(x_{0}\right)$ is not empty. Moreover,

$$
\max _{\lambda \in \Lambda_{a},|\lambda|=1} \frac{\partial^{2} \mathcal{L}}{\partial x^{2}}\left(x_{0}, \lambda\right)[h]^{2} \geq 0 \forall h \in \mathcal{K}\left(x_{0}\right) .
$$

We prove the theorem in three steps. First of all, assuming that $X$ is finitedimensional, we consider an auxiliary problem for which we obtain necessary conditions for a minimum. At the second step, using results obtained earlier, we prove the theorem in the case when $X$ is finite-dimensional. Finally, the proof of the theorem will be completed with the third step, at which we get rid of the assumption $\operatorname{dim} X<\infty$.

Step 1. Assume that $X$ is finite-dimensional. Let $\varphi$ be a given smooth scalar function on $X$ such that

$$
\frac{\partial \varphi}{\partial x}(x) \neq 0 \forall x: \varphi(x)=0 .
$$

We consider the auxiliary problem

$$
f(x) \rightarrow \min , x \in X, F(x) \in C, \varphi(x) \leq 0 .
$$

We suppose that $x_{0}$ is the solution. Let us prove that there exist Lagrange multipliers $\tilde{\lambda}=\left(\lambda^{0}, y, \beta\right) \neq 0$ and a subspace $\Pi \subset X$ such that

$$
\begin{gathered}
\frac{\partial \tilde{\mathcal{L}}}{\partial x}\left(x_{0}, \tilde{\lambda}\right)=0, \beta \varphi\left(x_{0}\right)=0, \lambda^{0} \geq 0, \beta \geq 0, y \in N_{C}\left(F\left(x_{0}\right)\right) ; \\
\operatorname{codim} \Pi=k+1 ; \Pi \subseteq \operatorname{Ker} F^{\prime}\left(x_{0}\right) \cap \operatorname{Ker}\left(\varphi^{\prime}\left(x_{0}\right)\left(1+\operatorname{sgn} \varphi\left(x_{0}\right)\right) ;^{2}\right. \\
\frac{\partial^{2} \tilde{\mathcal{L}}}{\partial x^{2}}\left(x_{0}, \tilde{\lambda}\right)[x]^{2} \geq 0 \forall x \in \Pi .
\end{gathered}
$$

Here $\tilde{\mathcal{L}}(x, \tilde{\lambda})=\lambda^{0} f(x)+\langle F(x), y\rangle+\beta \varphi(x)$.

Let us get rid of the constraints $F(x) \in C$ in the problem (3.4) with the help of the penalty method. We denote the distance from a point $y$ to the set $D$ by $\rho(y)$.

\footnotetext{
${ }^{2} \operatorname{sgn} \xi=-1 \forall \xi<0 ; \operatorname{sgn} 0=0$.
} 
The function $\rho$ is convex. Smoothing it, we can obtain the sequence of smooth functions $\left\{\rho_{i}\right\}$ :

$$
\rho_{i}(y)=0 \forall y \in C ; \rho_{i} \rightrightarrows \rho, i \rightarrow \infty ; \rho_{i}^{\prime}(y) \rightarrow \partial \rho(y), i \rightarrow \infty \forall y .
$$

Here $\partial \rho$ is the sub-differential of $\rho$ and the converging of the sequence of sets means that for any neighbourhood $O$ of the set $\partial \rho(y)$ there exists a number $i_{0}$ such that all the points $\rho_{i}^{\prime}(y)$ with the numbers $i \geq i_{0}$ belong to $O$. For each positive integer $i$ we set $f_{i}(x)=f(x)+i \rho_{i}(F(x))+\left|x-x_{0}\right|^{4}$. We shall consider the family of minimization problems

$$
f_{i}(x) \rightarrow \min , \varphi(x) \leq 0,\left|x-x_{0}\right| \leq \delta,
$$

called $i$-problems. Here $\delta>0$ was chosen so that $x_{0}$ would realise the minimum for problem (1.1) in the $\delta$-neighborhood of the point $x_{0}$. The solution of the $i$-problem is denoted by $x_{i}$.

Let us prove that $x_{i} \rightarrow x_{0}, i \rightarrow \infty$. Taking into account the finite dimensionality of $X$, we choose a subsequence of $\left\{x_{i}\right\}$ converging to a point $\bar{x}$. We shall show that $\bar{x}=x_{0}$.

Indeed,

$$
\begin{gathered}
f_{i}\left(x_{i}\right) \leq f_{i}\left(x_{0}\right)=f\left(x_{0}\right) \forall i \Rightarrow \rho_{i}\left(F\left(x_{i}\right)\right) \rightarrow 0, i \rightarrow \infty \\
\Rightarrow F(\bar{x}) \in C \Rightarrow f(\bar{x}) \geq f\left(x_{0}\right) .
\end{gathered}
$$

Besides, from the first inequality we have

$$
f\left(x_{i}\right)+\left|x_{i}-x_{0}\right|^{4} \leq f\left(x_{0}\right) \forall i \Rightarrow f(\bar{x})+\left|\bar{x}-x_{0}\right|^{4} \leq f\left(x_{0}\right) \leq f(\bar{x}) \Rightarrow \bar{x}=x_{0} .
$$

Thus $x_{i} \rightarrow x_{0}, i \rightarrow \infty$. For large numbers $i$, which are the only numbers we are going to consider, $\left|x_{i}-x_{0}\right|<\delta$. Hence the known first- and second-order necessary conditions for the $i$-problem are as follows [6]: there exist Lagrange multipliers $\tilde{\beta}_{i} \geq 0$ such that

$$
\left\{\begin{array}{c}
f_{i}^{\prime}\left(x_{i}\right)+\tilde{\beta}_{i} \varphi^{\prime}\left(x_{i}\right)=0 ; \tilde{\beta}_{i} \varphi\left(x_{i}\right)=0 \\
\left(f_{i}^{\prime \prime}\left(x_{i}\right)+\tilde{\beta}_{i} \varphi^{\prime \prime}\left(x_{i}\right)\right)[h]^{2} \geq 0 \forall h \in X: \sigma_{i}\left\langle h, \varphi^{\prime}\left(x_{i}\right)\right\rangle=0,
\end{array}\right.
$$

where $\sigma_{i}=1+\operatorname{sgn} \varphi\left(x_{i}\right)$.

Let us explain those conditions, setting

$$
\lambda_{i}^{\circ}=\left(1+\left|\tilde{\beta}_{i}\right|^{2}+\left|i \rho_{i}^{\prime}\left(F\left(x_{i}\right)\right)\right|^{2}\right)^{-1 / 2}, \beta_{i}=\tilde{\beta}_{i} \lambda_{i}^{\circ} ; y_{i}=i \rho_{i}^{\prime}\left(F\left(x_{i}\right)\right) \lambda_{i}^{\circ} .
$$

According to (3.7), we obtain

$$
\begin{gathered}
\lambda_{i}^{\circ} f^{\prime}\left(x_{i}\right)+\beta_{i} \varphi^{\prime}\left(x_{i}\right)+F^{\prime}\left(x_{i}\right)^{*} y_{i}+\lambda_{i}^{\circ} \times o\left(\left|x_{i}-x_{0}\right|\right)=0 ; \\
\left(\lambda_{i}^{\circ} f^{\prime \prime}\left(x_{i}\right)+\beta_{i} \varphi^{\prime \prime}\left(x_{i}\right)+F^{\prime \prime}\left(x_{i}\right) y_{i}\right)[h]^{2} \\
+i \lambda_{i}^{\circ} \rho_{i}^{\prime \prime}\left(F\left(x_{i}\right)\right)\left[F^{\prime}\left(x_{i}\right) h\right]^{2}+\lambda_{i}^{\circ} \times 1(i)[h]^{2} \geq 0 \\
\forall h \in X: \sigma_{i}\left\langle h, \varphi^{\prime}\left(x_{i}\right)\right\rangle=0 .
\end{gathered}
$$

Here $1(i)$ is a sequence converging to zero as $i \rightarrow \infty$. Set $\tilde{\lambda}_{i}=\left(\lambda_{i}^{\circ}, \beta_{i}, y_{i}\right)$. It is clear that $\left|\tilde{\lambda}_{i}\right|=1$. Then, passing to a suitable subsequence, we get $\tilde{\lambda}_{i} \rightarrow \tilde{\lambda}=$ $\left(\lambda^{\circ}, \beta, y\right), i \rightarrow \infty$, where $\tilde{\lambda} \neq 0, \lambda^{\circ} \geq 0, \beta \geq 0, \beta \varphi\left(x_{0}\right)=0$ and $y \in N_{C}\left(F\left(x_{0}\right)\right)$, for $y_{i}=i \rho_{i}^{\prime}\left(F\left(x_{i}\right)\right) \lambda_{i}^{\circ} \rightarrow \partial \rho\left(F\left(x_{0}\right)\right), i \rightarrow \infty$, by construction. As is known, for $y \in C$ the cone stretched over $\partial \rho(y)$ coincides exactly with $N_{C}(y)$ [7, p. 90]. Taking account of it and passing to the limit as $i \rightarrow \infty$ in (3.8), we obtain (3.5).

Let us prove the existence of a subspace $\Pi$ satisfying (3.6). For this we shall consider the sequence of the linear operators $A_{i}: X \rightarrow R^{k+1}$ defined by the formula 
$A_{i} x=\left(F^{\prime}\left(x_{i}\right) x, \sigma_{i}\left\langle\varphi^{\prime}\left(x_{i}\right), x\right\rangle\right) \forall x \in X$. On account of Theorem 2.1, there exists a subspace $\Pi$ in $X$ such that $\Pi \subseteq \operatorname{Ker} F^{\prime}\left(x_{0}\right) \cap \operatorname{Ker}\left(\sigma_{0} \varphi^{\prime}\left(x_{0}\right)\right) ; \Pi \subseteq L s \operatorname{Ker} A_{i}$ with codim $\Pi \leq k+1$. Consider an arbitrary $h \in \Pi$. Then, by the definition of the upper topological limit, there exist $h_{i} \in \operatorname{Ker} F^{\prime}\left(x_{i}\right) \cap \operatorname{Ker}\left(\sigma_{i} \varphi^{\prime}\left(x_{i}\right)\right)$ such that, passing to a suitable subsequence, we obtain $h_{i} \rightarrow h, i \rightarrow \infty$. Substituting the vectors $h_{i}$ for $h$ in (3.9) and taking the limit as $i \rightarrow \infty$, we get (3.6).

Remark. If $\varphi\left(x_{0}\right)<0$, then one can choose a subspace $\Pi$ satisfying (3.6) so that $\operatorname{codim} \Pi=k$. Indeed, if $\varphi\left(x_{0}\right)<0$, then $\sigma_{i}=\sigma_{0}=0$. Setting $A_{i}=F^{\prime}\left(x_{i}\right)$, we get the required subspace $\Pi$ with the help of Theorem 2.1.

Step 2. Let us assume, as before, that $X$ is finite-dimensional. From the reasoning in the first step it follows that $\Lambda_{a} \neq 0$ (because $x_{0}$ is the solution of the problem (3.4), where $\varphi$ is such that $\left.\varphi\left(x_{0}\right)<0 \Rightarrow \beta=0\right)$.

Let us prove (3.3). We shall use the method proposed by A. A. Agrachev in [8, p. 93]. It is convenient to suppose that $x_{0}=0, f\left(x_{0}\right)=0$, and $F\left(x_{0}\right)=0$. Fix an arbitrary $h \in \mathcal{K}$ with $|h|=1$.

For each $\varepsilon=i^{-1}, i=1,2, \ldots$, we shall consider the minimization problem

$$
\begin{cases}f_{\varepsilon}(x, \chi) \rightarrow \min , & 0 \leq \chi \leq 1 \\ F(x)-\chi F(\varepsilon h) \in C, & |x|^{2} \leq \delta^{2}\end{cases}
$$

over $X \times R^{1}$. Here $\delta>0$ was defined at the first step, and

$$
f_{\varepsilon}(x, \chi)=\tilde{f}(x)+|x-\varepsilon h|^{4}-\chi \tilde{f}(\varepsilon h) ; \tilde{f}(x)=f(x)+|x|^{4} .
$$

The solution of the problem (3.10) exists because the point $x=\varepsilon h, \chi=1$ satisfies all the constraints and the ball $\{x:|x| \leq \delta\}$ is compact. Let us denote the solution of $(3.10)$ by $\left(x_{i}, \chi_{i}\right)$.

We shall show that $\chi_{i}>0 \forall i$. Indeed, $f_{\varepsilon}(\varepsilon h, 1)=0 \Rightarrow f_{\varepsilon}\left(x_{i}, \chi_{i}\right) \leq 0$. On the other hand, in the case $\chi=0$ for $x$ such that $|x| \leq \delta$ and $F(x) \in C$ we have

$$
f_{\varepsilon}(x, 0)=f(x)+|x|^{4}+|x-\varepsilon h|^{4} \geq|x|^{4}+|x-\varepsilon h|^{4}>0 .
$$

Consequently, the point $(x, 0)$ is not the solution of (3.10). Therefore $\chi_{i}>0 \forall i$.

Besides,

$$
f_{\varepsilon}\left(x_{i}, \chi_{i}\right) \leq 0 \Rightarrow \tilde{f}\left(x_{i}\right)+\left|x_{i}-\varepsilon h\right|^{4} \leq|\tilde{f}(\varepsilon h)|, \varepsilon=i^{-1} \forall i
$$

then for any limit point $\hat{x}$ of the sequence $\left\{x_{i}\right\}$ the conditions $\tilde{f}(\hat{x}) \leq 0$ and $F(\hat{x}) \in C$ hold. Hence, $\hat{x}=0 \Rightarrow x_{i} \rightarrow 0, i \rightarrow \infty$.

Provided $i$ is sufficiently large, we apply the necessary minimum conditions obtained in the first step for the auxiliary problem (3.4) to the solution $\left(x_{i}, \chi_{i}\right)$ of the problem (3.10). We write them out, setting $\varphi(x, \chi) \equiv \chi-1$. We shall begin with the case when $\chi_{i}=1$ for every large $i$.

Determine the Lagrange function

$$
\tilde{\mathcal{L}}_{\varepsilon}\left(\lambda^{0}, \beta, y, x, \chi\right)=\lambda^{0} f_{\varepsilon}(x, \chi)+\beta(\chi-1)+\langle y, F(x)-\chi F(\varepsilon h)\rangle .
$$

The results of the first step show that there exist

$$
\lambda_{i}^{0} \geq 0, \beta_{i} \geq 0, y_{i} \in N_{C}\left(F\left(x_{i}\right)\right),
$$

which are not all equal to zero, and a subspace $\tilde{\Pi}_{i} \subseteq X \times R^{1}$ such that

$$
\begin{gathered}
\lambda_{i}^{0} \tilde{f}(\varepsilon h)+\left\langle y_{i}, F(\varepsilon h)\right\rangle=\beta_{i} \geq 0 ; \\
\lambda_{i}^{0}\left(f^{\prime}\left(x_{i}\right)+1(i)\right)+F^{\prime}\left(x_{i}\right)^{*} y_{i}=0 ;
\end{gathered}
$$




$$
\begin{gathered}
F^{\prime}\left(x_{i}\right) x-F(\varepsilon h) \chi=0, \chi=0 \forall \xi=(x, \chi) \in \tilde{\Pi}_{i} ; \\
\frac{\partial^{2} \tilde{\mathcal{L}}_{\varepsilon}}{\partial(x, \chi)^{2}}[\xi]^{2} \geq 0 \forall \xi \in \tilde{\Pi}_{i} ; \operatorname{codim} \tilde{\Pi}_{i} \leq k+1 .
\end{gathered}
$$

Note that the equality (3.11) is equivalent to the condition $\frac{\partial \tilde{\mathcal{L}}_{\varepsilon}}{\partial \chi}=0,(3.12)$ to the condition $\frac{\partial \tilde{\mathcal{L}}_{\varepsilon}}{\partial x}=0$ and (3.13) is a consequence of the fact that

$$
\left.\tilde{\Pi}_{i} \subseteq \operatorname{Ker} \frac{\partial}{\partial(x, \chi)}(F(x)-\chi F(\varepsilon h))\right|_{x=x_{i}, \chi=\chi_{i}} \cap \operatorname{Ker} \varphi^{\prime},
$$

(we recall that $\varphi \equiv \chi-1$ ).

We now analyze the formulas obtained. Set $\Pi_{i}=\left\{x \in X:(x, 0) \in \tilde{\Pi}_{i}\right\}$. Then, by (3.13),

$$
\Pi_{i} \subseteq \operatorname{Ker} F^{\prime}\left(x_{i}\right) \forall i
$$

and also codim $\Pi_{i} \leq k$. Moreover, by construction, the function $\tilde{\mathcal{L}}_{\varepsilon}$ is linear with respect to the variable $\chi$. Therefore the quadratic form $\partial^{2} \tilde{\mathcal{L}}_{\varepsilon} / \partial x^{2}$ is nonnegative on $\Pi_{i}$. Furthermore, by Theorem 2.2 there exists a subspace $\Pi \subseteq X$ such that $\operatorname{codim} \Pi \leq k$ and $\Pi \subseteq L s\left\{\Pi_{i}\right\}$.

Let us pass to the limit as $i \rightarrow \infty$ in our formulas. Set $\lambda_{i}=\left(\lambda_{i}^{0}, y_{i}\right)$. From (3.13) it follows that $\lambda_{i} \neq 0$. Hence, after normalizing $\lambda_{i}$, we may assume $\left|\lambda_{i}\right|=1 \forall i$. Passing to a suitable subsequence, we obtain $\lambda_{i} \rightarrow \lambda=\left(\lambda^{0}, y\right), i \rightarrow \infty \Rightarrow \lambda^{0} \geq$ $0, \lambda \neq 0$. Besides, $y \in N_{C}\left(F\left(x_{0}\right)\right)$, for the normal cone $N_{C}(y)$ is semi-continuous from above with respect to $y \in C$. Thus we get (3.1) by passing to the limit as $i \rightarrow \infty$ in (3.12).

Let us prove that $\lambda \in \Lambda_{a}$. Indeed, consider an arbitrary $z \in \Pi$. Then there exists a sequence $\left\{z_{i}\right\}$ such that, after passing to a suitable subsequence, we have $z_{i} \in$ $\Pi_{i} \forall i: z_{i} \rightarrow z, i \rightarrow \infty$. Therefore, by (3.15), $z_{i} \in \operatorname{Ker} F^{\prime}\left(x_{i}\right) \forall i \Rightarrow z \in \operatorname{Ker} F^{\prime}\left(x_{0}\right)$. Consequently, $\Pi \subseteq \operatorname{Ker} F^{\prime}\left(x_{0}\right)$. By analogy, from (3.14) we get

$$
\frac{\partial^{2} \mathcal{L}}{\partial x^{2}}\left(x_{0}, \lambda\right)[z]^{2} \geq 0 \forall z \in \Pi \Rightarrow \lambda \in \Lambda_{a} .
$$

Return again to the inequalities (3.11). By assumption, $C$ is a convex cone with a finite number of faces. Hence $N_{C}(y) \subseteq N_{C}\left(F\left(x_{0}\right)\right)$ for all $y \in C$ close to $F\left(x_{0}\right)$. Consequently, $y_{i} \in N_{C}\left(F\left(x_{0}\right)\right)$ for large $i$. Also, $h \in \mathcal{K} \Rightarrow\left\langle y_{i}, F^{\prime}\left(x_{0}\right) h\right\rangle \leq 0$ and $\lambda_{i}^{0}\left\langle f^{\prime}\left(x_{0}\right), h\right\rangle \leq 0$. Using those inequalities and expanding the left part of (3.11) up to the members of the second order with respect to $\varepsilon$, passing to the limit as $i \rightarrow \infty$, we obtain $\frac{\partial^{2} \mathcal{L}}{\partial x^{2}}\left(x_{0}, \lambda\right)[h]^{2} \geq 0$. Hence, due to the arbitrariness of $h$, we get (3.3).

Let us note that if $\chi_{i}<1$ for an infinite number of $i$, the problem is considered in the same way. We have to use the remark given at the end of the first step.

Step 3. Let us prove the theorem in complete generality (i.e. in the case when $\operatorname{dim} X=\infty)$. We shall use the transition proposed in [9]. Let $x_{0}=0$. We consider an arbitrary $h \in \mathcal{K}$. Let $\mathcal{M}$ denote the set of the finite-dimensional subspaces $M \subset X$ that each contain $h$ and satisfy the condition $F^{\prime}\left(x_{0}\right)(M)=\operatorname{Im} F^{\prime}\left(x_{0}\right)$. Let us consider an arbitrary $M \in \mathcal{M}$ and examine the problem we get from (1.1) 
after replacing $X$ with $M$. The results of the second step show that for this finitedimensional problem there exist Lagrange multipliers $\lambda_{M}=\left(\lambda_{M}^{0}, y_{M}\right)$ such that

$$
\lambda_{M}^{0} \geq 0, y_{M} \in N_{C}\left(F\left(x_{0}\right)\right),\left|\lambda_{M}\right|=1 ; \frac{\partial \mathcal{L}}{\partial x}\left(x_{0}, \lambda_{M}\right) \in M^{\perp} ; \frac{\partial^{2} \mathcal{L}}{\partial x^{2}}\left(x_{0}, \lambda_{M}\right)[h]^{2} \geq 0,
$$

and the index of the quadratic form $\frac{\partial^{2} \mathcal{L}}{\partial x^{2}}\left(x_{0}, \lambda_{M}\right)$ on the subspace $M \cap \operatorname{Ker} F^{\prime}\left(x_{0}\right)$ does not exceed codim $\left(\operatorname{Im} F^{\prime}\left(x_{0}\right)\right)$. The set of such vectors $\lambda_{M}$ is denoted by $\Lambda_{a}(M, h)$. It is easy to see that the set $\Lambda_{a}(M, h)$ is closed and nonempty for any $M \in \mathcal{M}$. Moreover, it is obvious that

$$
\bigcap_{i=1}^{n} \Lambda_{a}\left(M_{i}, h\right) \supseteq \Lambda_{a}\left(M_{1}+\ldots+M_{n}, h\right) \neq \emptyset
$$

for an arbitrary $M_{1}, \ldots, M_{n} \in \mathcal{M}$. This is, the system of sets $\Lambda_{a}(M, h), M \in \mathcal{M}$, has the finite intersection property. Consequently, since the unit sphere in $R^{k+1}$ is compact, the intersection $\bigcap_{M \in \mathcal{M}} \Lambda_{a}(M, h)$ is not empty. It is evident that for an arbitrary vector $\lambda \in \bigcap_{M \in \mathcal{M}} \Lambda_{a}(M, h)$

$$
\lambda \in \Lambda_{a} ; \frac{\partial^{2} \mathcal{L}}{\partial x^{2}}\left(x_{0}, \lambda\right)[h]^{2} \geq 0 .
$$

From this and the arbitrariness of $h \in K$ we get (3.3). The theorem is established.

Remark. The theorem stay true even if the condition that the cone $C$ has a finite number of faces is replaced by the following assumption:

$$
\exists \varepsilon>0: N_{C}(F(x)) \subseteq N_{C}\left(F\left(x_{0}\right)\right) \forall x: F(x) \in C,\left|F(x)-F\left(x_{0}\right)\right|<\varepsilon .
$$

That can be easily shown by analysing the proof of the theorem, for in it we did not use the fact that the cone $C$ has a finite number of faces, but only its consequence (3.16).

2. Now we consider the problem (1.1) assuming not that $x_{0}$ is simply abnormal but that "complete degeneracy" occurs at that point, i.e.

$$
f^{\prime}\left(x_{0}\right)=0 ; F^{\prime}\left(x_{0}\right)=0 .
$$

Although the "complete degeneracy" seems unnatural, there are problems that can be reduced to the case (3.17), for example, the problem of the fixed-sign property of a quadratic form on the intersection of a finite number of quadrics [10]. It turns out to be possible to complement the necessary conditions of Theorem 3.1 in the case of "complete degeneracy".

Theorem 3.2. Assume that $\operatorname{dim} X<\infty, C=0$ and (3.17) holds. Then there exist Lagrange multipliers $\lambda=\left(\lambda^{0}, y\right) \in \Lambda_{a}$ and a vector $l \in X$ such that

$$
\frac{\partial^{2} \mathcal{L}}{\partial x^{2}}\left(x_{0}, \lambda\right) l=0 ; y=\frac{\partial^{2} F}{\partial x^{2}}\left(x_{0}\right)[l]^{2},
$$

$$
\frac{\partial^{2} f}{\partial x^{2}}\left(x_{0}\right)[l]^{2} \leq 0 ; \frac{\partial^{2} \mathcal{L}}{\partial x^{2}}\left(x_{0}, \lambda\right)[x]^{2}+2\left|\frac{\partial^{2} F}{\partial x^{2}}\left(x_{0}\right)[l, x]\right|^{2} \geq 0 \forall x \in X ; \lambda^{0}+|l| \neq 0 .
$$

Proof. We shall use the reasoning from the first step of Theorem 3.1. It is convenient to suppose that $x_{0}=0, f(0)=0$. We consider the auxiliary problem (3.4) for $C=0, \varphi \equiv-1$. Construct the family of $i$-problems, setting $\rho_{i}(y) \equiv \rho(y) \equiv|y|^{2}$. 
If for an infinite number of $i$ the solutions $x_{i}$ of $i$-problems are zero, then the assertion of the theorem with $\lambda^{0}=1, y=0, l=0$ follows from (3.9). Therefore we are left to consider the case $x_{i} \neq 0 \forall i$.

Let us set

$$
\gamma_{i}=\left(1+i^{2}\left|x_{i}\right|^{4}\right)^{\frac{1}{2}}, l_{i}=\gamma_{i}^{-\frac{1}{2}} i^{\frac{1}{2}} x_{i}
$$

It is evident that

$$
f_{i}\left(x_{i}\right) \leq 0 \Rightarrow f\left(x_{i}\right) \leq 0 \forall i
$$

From this and (3.17), $f^{\prime \prime}\left(x_{0}\right)\left[l_{i}\right]^{2} \leq 1(i)$.

According to (3.17), we get

$$
\begin{gathered}
f^{\prime}\left(x_{i}\right)=f^{\prime \prime}(0) x_{i}+o\left(\left|x_{i}\right|\right), F(x)=\frac{1}{2} F^{\prime \prime}(0)[x]^{2}+o\left(|x|^{2}\right), \\
F^{\prime}\left(x_{i}\right)=F^{\prime \prime}(0) x_{i}+o\left(\left|x_{i}\right|\right) .
\end{gathered}
$$

After substituting those representations in (3.8) and (3.9) we divide the former by $\lambda_{i}^{0} \gamma_{i} \gamma_{i}^{\frac{1}{2}} i^{-\frac{1}{2}}$ and the latter by $\lambda_{i}^{0} \gamma_{i}$. Taking into consideration that $\rho^{\prime}(y)=2 y$, $\rho^{\prime \prime}(y)=2 I$ ( $I$ is a unit matrix) and passing to the limit as $i \rightarrow \infty$, we obtain the required $\lambda$ and $l$. The theorem is proved.

Remark. It follows from the example given below that the assumption on the finitedimensionality of $X$ is fundamental in Theorem 3.2-it cannot be omitted without being replaced by another one (for example, by the assumption that the form is of the Legendre type, as was done in $[5,11])$.

Example. Let $X$ be a Hilbert space, $A: X \rightarrow X$ a positive symmetric compact operator and $I: X \rightarrow X$ an identity operator. Then zero is the solution of the problem

$$
f(x)=-|x|^{2} \rightarrow \min ; F(x)=\langle A x, x\rangle=0,
$$

for $A[x]^{2} \neq 0 \forall x \neq 0$. Let us assume that $\lambda^{0} \geq 0, y \in R^{1}, l \in X$ satisfy the conditions of Theorem 3.2. Then the index of the form $\left(-\lambda^{0} I+y A\right)$ does not exceed unity. Hence $\lambda^{0}=0$, for $A$ is compact. Therefore $y \neq 0 \Rightarrow l=0$, because $y A l=0$, but $\operatorname{Ker} A=0$. Thus, we have a contradiction.

Definition $([12])$. The mapping $F$ is called $2-$ regular at a point $x_{0}$ if

$$
\begin{gathered}
\operatorname{Im}\left(F^{\prime}\left(x_{0}\right)+P F^{\prime \prime}\left(x_{0}\right) x\right)=Y \\
\forall x: F^{\prime}\left(x_{0}\right) x=0, F^{\prime \prime}\left(x_{0}\right)[x, x] \in \operatorname{Im} F^{\prime}\left(x_{0}\right) .
\end{gathered}
$$

Here $P$ is the operator of orthogonal projection from $Y$ onto $\left(\operatorname{Im} F^{\prime}\left(x_{0}\right)\right)^{\perp}$.

Theorem 3.3. Assume that $\operatorname{dim} X<\infty$ and at least one of the following assumptions holds: either $F^{\prime}\left(x_{0}\right)=0$ and the mapping $F$ is 2-regular at the point $x_{0}$, or $x_{0}=0$ and $F$ is a quadratic mapping. Let $f^{\prime}\left(x_{0}\right)=0$ and

$$
f(x) \geq f\left(x_{0}\right)+\varepsilon\left|x-x_{0}\right|^{2} \forall x: F(x)=0,\left|x-x_{0}\right|<\varepsilon
$$

for $\varepsilon>0$. Then there exist $\lambda \in \Lambda_{a}, l \in X$ satisfying (3.18) and (3.19) such that $\lambda^{0}>0$.

The validity of this statement follows easily from Theorem 3.2 and Theorem 2 of $[12$, p. 1095].

3. For the first time second-order necessary conditions that require the set $\Lambda_{a}\left(x_{0}\right)$ to be nonempty were obtained for the time optimality problem in [13]. Further, 
those conditions were generalized to a broad class of extremal and optimal control problems $[5,9,11]$.

A. A. Agrachev [14] was the first to obtain necessary conditions under the assumption that the combined mapping $\varphi=(f, F)$ satisfies a condition similar to 2 -regularity at $x_{0}$ and $\varphi^{\prime}\left(x_{0}\right)=0$. Those conditions can be easily derived from (3.3). For this we should first replace the number $k$ in (3.2) with $k+1$ to obtain the set $\tilde{\Lambda}$ of the corresponding Lagrange multipliers (therefore $\Lambda_{a} \subset \tilde{\Lambda}$ ) and then replace the set $\Lambda_{a}$ in (3.3) by the set $\tilde{\Lambda}$. In the same paper it was proved that those conditions (under the same assumptions) are also sufficient.

Second-order necessary conditions with supremum taken over a subset of the cone $\Lambda$ of the Lagrange multipliers were obtained by A. A. Milyutin in $[15,16]$. Namely, he proved that

$$
\Lambda_{+} \neq 0 ; \sup _{\lambda \in \Lambda_{+},|\lambda|=1} \frac{\partial^{2} \mathcal{L}}{\partial x^{2}}\left(x_{0}, \lambda\right)[x]^{2} \geq 0 \forall x \in \mathcal{K}\left(x_{0}\right) .
$$

Here $\Lambda_{+}$is the set of those $\lambda \in \Lambda$ for which the index of the quadratic form $\frac{\partial^{2} \mathcal{L}}{\partial x^{2}}\left(x_{0}, \lambda\right)$ is finite. It is clear that the necessary conditions (3.21) are weaker than those obtained in Theorem 3.1 (a problem for which (3.21) holds, but $\Lambda_{a}\left(x_{0}\right)=\emptyset$ and the assertion of Theorem 3.1 is therefore not true, and consequently $x_{0}$ is not a local minimum, can be found in [5, p. 190]). Moreover, if $X$ is finite-dimensional, then $\Lambda_{+}=\Lambda$ and it is easy to see that the conditions (3.21) hold automatically at an abnormal point (see (1.3)), i.e. do not depend on the objective function $f$.

New and significant necessary optimality conditions for extremal problems in the abnormal case were obtained by E. R. Avakov in [17, 23]. To deduce them he introduced a modified Lagrange function and proved that it satisfies the Lagrange equation and its second-order derivative was nonnegative for each $x$ satisfying (3.20) and belonging to the cone

$$
x: F^{\prime}\left(x_{0}\right) x=0, F^{\prime \prime}\left(x_{0}\right)[x, x] \in \operatorname{Im} F^{\prime}\left(x_{0}\right) .
$$

We compare Theorem 3.1 with Avakov's results below (see the end of $\S 4$ ).

\section{THE NOTION OF 2-NORMALITY \\ AND SECOND-ORDER SUFFICIENT CONDITIONS}

As mentioned earlier, one of the most important characteristics of second-order extremum conditions is the value of the gap between their property of being necessary and that of being sufficient. It seems natural to regard this gap as minimal as possible with respect to certain second-order necessary conditions if they turn into sufficient ones after sufficiently small (in the $C^{2}$ metric) perturbations of the objective function and the mapping representing constraints. "Sufficiently small perturbations" means perturbations which would not change the values of $f, F$ and their first derivatives at the point under consideration. Our aim is to make clear when this gap is as minimal as possible with respect to the second-order necessary conditions obtained earlier $(\S 3)$. In order to do this, let us first deduce sufficient conditions.

Assume that $x_{0} \in X$, and $f$ and $F$ are twice continuously differentiable in a neighborhood of $x_{0}$ with respect to the finite topology.

Theorem 4.1. Let the following conditions hold for the point $x_{0}$ :

(i) $\Lambda_{a}=\Lambda_{a}\left(x_{0}\right) \neq \emptyset$, 
(ii) $\max _{\lambda \in \Lambda_{a},|\lambda|=1} \frac{\partial^{2} \mathcal{L}}{\partial x^{2}}\left(x_{0}, \lambda\right)[x]^{2}>0 \forall x \in \mathcal{K}\left(x_{0}\right): x \neq 0$.

Then $x_{0}$ is a strict local minimum (with respect to the finite topology $\tau$ ) for the problem (1.1).

Proof. Let us consider a $\tau$-neighbourhood $V$ of the point $x_{0}$ where $f$ and $F$ are twice continuously differentiable. By $\tilde{O}$ we denote the set of those points $x \in V$ for which either $F(x) \notin C$, or $F(x) \in C, f(x)>f\left(x_{0}\right)$. Set $O=\tilde{O} \cup\left\{x_{0}\right\}$. Obviously, it is enough to prove that $O$ is a $\tau$-neighbourhood of the point $x_{0}$. Indeed, let $M \subset X$ be an arbitrary finite-dimensional subspace that contains $x_{0}$. We shall prove that $M \cap O$ is open in $M$.

To begin with, let us show that $M \cap \tilde{O}$ is open in $M$. Let $\bar{x} \in M \cap \tilde{O}$. Then either $F(\bar{x}) \notin C$, or $f(\bar{x})>f\left(x_{0}\right)$. Since the restrictions of $F$ and $f$ to $V \cap M$ are continuous and $C$ is closed, the point $\bar{x}$ possesses a neighbourhood $W$ in $M$ such that either $F(W) \cap C=\emptyset$, or $f(x)>f\left(x_{0}\right) \forall x \in W$. This proves that $M \cap \tilde{O}$ is open in $M$.

Let us examine the finite-dimensional problem

$$
f(x) \rightarrow \min , x \in M, F(x) \in C .
$$

From the assumptions of the theorem and the finite-dimensionality of $M$ it follows that for this problem the sufficient conditions [15] for a strict local minimum holds for the point $x_{0}$. Hence there exists a neighbourhood of $x_{0}$ in $M$ such that for every $x \neq x_{0}$ of this neighbourhood with $F(x) \in C$ the inequality $f(x)>f\left(x_{0}\right)$ is true. From this and the fact that $M \cap \tilde{O}$ is open in $M$, it follows that $M \cap O$ is open in $M$. By the arbitrariness of the subspace $M$, the set $O$ is a $\tau$-neighbourhood of the point $x_{0}$. And by construction, we have

$$
x \in O, F(x) \in C, x \neq x_{0} \Rightarrow f(x)>f\left(x_{0}\right) .
$$

Consequently, $x_{0}$ is a strict local minimum for the problem (1.1). The theorem is established.

It is natural that a minimum stronger that the one in the finite topology requires stronger assumptions.

Let $X$ be a Banach space. We shall assume that $f$ and $F$ are twice continuously differentiable in a neighbourhood of $x_{0}$ and there exists a functional $\gamma$ strictly differentiable at $x_{0}$ such that $\gamma(0)=0$ and $\gamma(x)>0 \forall x \neq 0 . \gamma$ is called a strict highest order [15] and satisfies the following conditions:

$$
\begin{aligned}
& \left\|f(x)-\left(f\left(x_{0}\right)+\left\langle f^{\prime}\left(x_{0}\right), x-x_{0}\right\rangle+\frac{1}{2} f^{\prime \prime}\left(x_{0}\right)\left[x-x_{0}\right]^{2}\right)\right\|=o\left(\gamma\left(x-x_{0}\right)\right), \\
& \left\|F(x)-\left(F\left(x_{0}\right)+F^{\prime}\left(x_{0}\right)\left(x-x_{0}\right)+\frac{1}{2} F^{\prime \prime}\left(x_{0}\right)\left[x-x_{0}\right]^{2}\right)\right\|=o\left(\gamma\left(x-x_{0}\right)\right) .
\end{aligned}
$$

Theorem $4.2([15])$. Let $\Lambda_{a} \neq \emptyset$ and

$$
\max _{\lambda \in \Lambda_{a},|\lambda|=1} \frac{\partial^{2} \mathcal{L}}{\partial x^{2}}\left(x_{0}, \lambda\right)[x]^{2} \geq \gamma(x) \forall x \in \mathcal{K}\left(x_{0}\right) .
$$

Then $x_{0}$ is a strict local minimum for the problem (1.1). 
This theorem is a trivial consequence of the results of $[15, \S 8]$ for $\Lambda_{a} \subseteq \Lambda\left(x_{0}\right)$. Let us note that in [15] the authors build a general theory of sufficient conditions.

Now we can make clear when the gap between the second-order necessary $(\S 3)$ and sufficient conditions is as minimal as possible. It turns out that everything depends on whether the cone conv $\Lambda_{a}\left(x_{0}\right)$ is pointed ${ }^{3}$ or not. The fact is that the maximum over the set $\left\{\lambda \in \Lambda_{a}:|\lambda|=1\right\}$ in (3.3) coincides with the maximum over the convex hull conv $\Lambda_{a}$. Thus, if the cone conv $\Lambda_{a}$ is not pointed (and, consequently, contains $\lambda=\left(\lambda^{0}, y\right)$ such that $\left.(-\lambda) \in \operatorname{conv} \Lambda_{a}\right)$, then it is obvious that the condition (3.3) holds. In that case (3.3) holds for any objective function $f$, and therefore it does not provide us with any information useful for the minimization problem (1.1). Thus, if the cone conv $\Lambda_{a}$ is not pointed, one should not hope, generally speaking, that after sufficiently small (in the $C^{2}$ metric) perturbations of $F$ and $f$ the point $x_{0}$ or any point close to it would realise a local minimum in the perturbed problem. Here "sufficiently small perturbations" means those that do not change the values of $f\left(x_{0}\right), F\left(x_{0}\right), f^{\prime}\left(x_{0}\right), F^{\prime}\left(x_{0}\right)$. It is substantiated by the following example:

$$
\begin{gathered}
X=R^{n}, Y=R^{1}, C=0, f(x)=-|x|^{2} \rightarrow \min ; \\
F(x)=x_{1} x_{2}=0, x=\left(x_{1}, \ldots, x_{n}\right) .
\end{gathered}
$$

The cone $\Lambda_{a}=\Lambda_{a}(0)$ at the point $x_{0}=0$ is not empty, and conv $\Lambda_{a}$ is not pointed. Hence, the conditions (3.3) hold for zero. Nevertheless, it follows from Theorem 3.2 that $x_{0}$ cannot be a local minimum for any perturbed problem obtained from the original by means of arbitrarily small (in the $C^{2}$ metric) smooth perturbations of $f$ and $F$ such that $F(0)=F^{\prime}(0)=0$.

If the cone conv $\Lambda_{a}$ is pointed, the situation is completely different.

For $x \in X$ let $\mathcal{F}_{2}(x)$ denote the cone consisting of $y \in Y, \quad y \neq 0$ such that $y \in N_{C}(F(x))$ and $\left(F^{\prime}(x)\right)^{*} y=0$. There exists a closed subspace $\Pi \subseteq \operatorname{Ker} F^{\prime}(x)$ in $X$ such that codim $\Pi \leq k ; \frac{\partial^{2}}{\partial x^{2}}\langle F(x), y\rangle[h]^{2} \geq 0 \forall h \in \Pi$.

Definition. The mapping $F$ is called 2-normal with respect to the cone $C$ at a point $x$ if the cone conv $\mathcal{F}_{2}(x)$ is pointed (we do not exclude the case $\mathcal{F}_{2}(x)=\emptyset$, because the empty cone is pointed by definition).

That definition is geometric and not useful for checking the 2-normality property. Hence, we give in $\S 5$ a criterion for 2-normality and study the properties of 2-normal mappings. Here we only remark that for a smooth function $f$ the mapping $F$ being 2-normal with respect to $C$ at a point $x$ is equivalent to the cone conv $\Lambda_{a}(x)$ being pointed.

Let us assume that $X$ is a Banach space and there exists a function $\Gamma$ such that $\Gamma$ is twice continuously differentiable in a neighbourhood of a point $x_{0}$ and $\Gamma\left(x_{0}\right)=0, \quad \Gamma^{\prime}\left(x_{0}\right)=0$. Let the functional $\gamma(x)=\Gamma^{\prime \prime}\left(x_{0}\right)[x]^{2}$ be a highest order (i.e. $\gamma$ is strictly differentiable and $\gamma(x)>0 \forall x \neq 0$ ). Besides, the mappings $f$ and $F$ are assumed to be twice continuously differentiable in a neighbourhood of $x_{0}$ and to satisfy the equalities (4.1), (4.2).

Theorem 4.3. Assume that the mapping $F$ is 2-normal with respect to the cone $C$ at the point $x_{0}$ and the following second-order necessary conditions hold for this

\footnotetext{
${ }^{3} \mathrm{~A}$ convex cone is called pointed if it does not contain nontrivial subspaces. We will also agree to regard the empty set as a pointed cone.
} 
point:

$$
\Lambda_{a}=\Lambda_{a}\left(x_{0}\right) \neq \emptyset ; \max _{\lambda \in \Lambda_{a},|\lambda|=1} \frac{\partial^{2} \mathcal{L}}{\partial x^{2}}\left(x_{0}, \lambda\right)[x]^{2} \geq 0 \forall x \in \mathcal{K}\left(x_{0}\right) .
$$

Then there exists a vector $\bar{y} \in Y$ such that for any $\varepsilon>0$ the point $x_{0}$ is a strict local minimum for the perturbed problem

$$
\left\{\begin{array}{l}
f_{\varepsilon}(x)=f(x)+\varepsilon \Gamma(x) \rightarrow \min \\
F_{\varepsilon}(x)=F(x)+\varepsilon \Gamma(x) \bar{y} \in C .
\end{array}\right.
$$

Proof. Since the mapping $F$ is 2-normal, the cone conv $\Lambda_{a}$ is pointed, and so its conjugate cone has nonempty interior (because $\operatorname{dim} Y<\infty$ ). Let us choose an arbitrary vector $z=(\alpha, \bar{y}) \in \operatorname{int}\left(\operatorname{conv} \Lambda_{a}\right)^{*}$ with $|\alpha|<1$. Therefore $0<\varepsilon\langle(1, \bar{y}), \lambda\rangle$ $\forall \lambda \in \Lambda_{a}$. From this and (4.3) we see that all the assumptions of Theorem 4.2 hold for the perturbed problem. Consequently, $x_{0}$ is a strict local minimum for the perturbed problem. The theorem is proved.

Remark. If the order $\gamma$ is a quadratic form in the statement of Theorem 4.2, then it can be taken for the function $\Gamma$.

We return to Avakov's results $[17,23]$ to compare them with Theorem 3.1. Suppose that the mapping $F$ is 2 -normal at a point $x_{0}$ with respect to the cone $C$. Then the results of $[17,23]$ follow from $(3.3)$. That could be shown with the aid of Theorem 3.1 and 4.3. Moreover, Avakov's results do not give any useful information for the problem of the fixed-sign property of a quadratic form on the intersection of a finite number of quadrics [10], but Theorem 3.1 does. On the other hand, from Avakov's conditions $[17,23]$ it follows that the point $x_{0}=0$ is not a local minimum for the example mentioned above. In addition, in $[17,23]$ the case $\operatorname{dim} Y=\infty$ there was examined.

\section{Properties of 2-Normal MAPpings}

1. Let us consider a point $x_{0} \in X$ and set

$$
d=\operatorname{dim}\left(\operatorname{Im} F^{\prime}\left(x_{0}\right)\right)^{\perp} \cap N_{C}\left(F\left(x_{0}\right)\right) \cap\left(-N_{C}\left(F\left(x_{0}\right)\right)\right) .
$$

Lemma 5.1. The mapping $F$ is 2-normal with respect to $C$ at a point $x_{0}$ if and only if for any integer $s, 1 \leq s \leq d$, there do not exist linearly independent vectors $y_{i} \in Y, i=1, \ldots, s$, such that

$$
y_{i} \in \operatorname{Ker}\left(F^{\prime}\left(x_{0}\right)^{*}\right) \cap N_{C}\left(F\left(x_{0}\right)\right) \cap\left(-N_{C}\left(F\left(x_{0}\right)\right) ; i=1, \ldots, s,\right.
$$

$$
\operatorname{ind}_{Z} \frac{\partial^{2}}{\partial x^{2}}\left\langle y_{i}, F\left(x_{0}\right)\right\rangle \leq d, i=1, \ldots, s ; \operatorname{ind}_{Z}\left(-\sum_{i=1}^{s} \frac{\partial^{2}}{\partial x^{2}}\left\langle y_{i}, F\left(x_{0}\right)\right\rangle\right) \leq d .
$$

Here $Z=\operatorname{Ker} F^{\prime}\left(x_{0}\right)$.

Proof. $0 \notin \mathcal{F}_{2}\left(x_{0}\right)$ by definition. Hence, the cone conv $\mathcal{F}_{2}\left(x_{0}\right)$ is not pointed if, and only if, it contains zero. Let $0 \in \operatorname{conv} \mathcal{F}_{2}\left(x_{0}\right)$. Then by the Carathéodory theorem, there exist $y_{i} \in \mathcal{F}_{2}\left(x_{0}\right), i=1, \ldots, k+1$, which are not all zero, such that $\sum_{i=1}^{k+1} y_{i}=0$. Hence the $y_{i}, i=1, \ldots, k+1$, satisfy (5.2). Thus, applying the consequence of the Carathéodory theorem [18] to the system of vectors $\left\{y_{i}\right\}$, we reduce (after renumbering) the number of vectors to $s+1$ for a positive integer $s \leq d$ 
so that the vectors $y_{1}, \ldots, y_{s+1}$ are affinally independent and $\sum_{i=1}^{s+1} y_{i}=0$. Having renumbered this system, we obtain one with linearly independent vectors in the first $s$ places. Using the fact that $y_{s+1}=-\sum_{i=1}^{s} y_{i}, y_{i} \in \mathcal{F}_{2}\left(x_{0}\right) \forall i$, we get (5.3). If we reverse the reasoning, from the conditions (5.2), (5.3) we get $0 \in \operatorname{conv} \mathcal{F}_{2}\left(x_{0}\right)$. The lemma is proved.

By Lemma 5.1, if $x$ is normal then the mapping $F$ is 2-normal at that point, but not vice versa. It was proved in [8, p. 92] that if $X=R^{n}$ and $n \gg k$, then a typical quadratic mapping is 2-normal at zero.

2. Let us bring up sufficient conditions for 2-normality of the mapping with respect to the cone $C$. The first one requires the interior of $C$ not to be empty (i.e. there are only inequality-type constraints in the minimization problem). Then, evidently, $F$ is 2-normal at every point. The other sufficient conditions for 2normality are not so obvious. We shall formulate them.

Consider the symmetric bilinear mapping $\frac{\partial^{2} F}{\partial x^{2}}\left(x_{0}\right)$ defined on the subspace $\operatorname{Ker} F^{\prime}\left(x_{0}\right)$. For every $y \in Y$ it generates a symmetric bilinear form $Q=y \frac{\partial^{2} F}{\partial x^{2}}\left(x_{0}\right)$ by the formula

$$
Q\left(x_{1}, x_{2}\right)=\frac{\partial^{2}}{\partial x^{2}}\left(\left\langle y, F\left(x_{0}\right)\right\rangle\right)\left[x_{1}, x_{2}\right] \forall x_{1}, x_{2} \in \operatorname{Ker} F^{\prime}\left(x_{0}\right) .
$$

This bilinear form generates a linear operator $\bar{Q}: \operatorname{Ker} F^{\prime}\left(x_{0}\right) \rightarrow X^{*}$ by the formula $\langle\bar{Q} x, \xi\rangle=Q(x, \xi) \forall x \in \operatorname{Ker} F^{\prime}\left(x_{0}\right), \xi \in X$. Let us denote the bilinear form $y \frac{\partial^{2} F}{\partial x^{2}}\left(x_{0}\right)$ and the operator generated by it alike. Together with this we have

$$
\operatorname{Ker}\left(y \frac{\partial^{2} F}{\partial x^{2}}\left(x_{0}\right)\right)=\left\{z \in \operatorname{Ker} F^{\prime}\left(x_{0}\right):\left\langle y, \frac{\partial^{2} F}{\partial x^{2}}\left(x_{0}\right)[z, \xi]\right\rangle=0 \forall \xi \in \operatorname{Ker} F^{\prime}\left(x_{0}\right)\right\}
$$

by definition.

Theorem 5.1. Assume that for every positive integer $s \leq d$ and any linearly independent vectors $y_{i}, i=1, \ldots, s$, satisfying (5.2) the condition

$$
\operatorname{codim}\left(\bigcap_{i=1}^{s} \operatorname{Ker}\left(y_{i} \frac{\partial^{2} F}{\partial x^{2}}\left(x_{0}\right)\right)\right)>d(s+1)
$$

holds. Here codim is codimension in the subspace $\operatorname{Ker}^{\prime}\left(x_{0}\right)$. Then the mapping $F$ is 2-normal with respect to the cone $C$ at the point $x_{0}$.

3. Let us preface the proof of Theorem 5.1 with an algebraic result related to the study of the family of $n \times n$-symmetric matrices $Q_{i}, i=1, \ldots, s$, of a special kind ( $s, n$ are positive integers).

Let $n_{1}, n_{2}$ be positive integers and $n_{1}+n_{2}=n$. The matrices $Q_{i}$ are of the form

$$
Q_{i}=\left(\begin{array}{cc}
B_{i} & A_{i}^{*} \\
A_{i} & O
\end{array}\right), i=1, \ldots, s .
$$

Here $B_{i}$ are $n_{1} \times n_{1}$-symmetric matrices, $A_{i}$ are $n_{2} \times n_{1}$ rectangular matrices and $O$ is the $n_{2} \times n_{2}$ trivial matrix.

Lemma 5.2. Let $\Pi_{i}, i=0, \ldots, s$, be given subspaces from $R^{n_{1}}$, and $\Pi=\bigcap_{i=0}^{s} \Pi_{i}$. Assume that

$$
\Pi_{i} \subseteq \operatorname{Ker} A_{i}, i=1, \ldots, s ; \Pi_{0} \subseteq \operatorname{Ker}\left(\sum_{i=1}^{s} A_{i}\right)
$$




$$
\begin{gathered}
\left\langle B_{i} \bar{\xi}, \xi\right\rangle=0 \forall \xi \in \Pi, \bar{\xi} \in \Pi_{i}, i=1, \ldots, s ; \\
\left\langle\sum_{i=1}^{s} B_{i} \bar{\xi}, \xi\right\rangle=0 \forall \xi \in \Pi, \bar{\xi} \in \Pi_{0} .
\end{gathered}
$$

Then

$$
\operatorname{codim}\left(\bigcap_{i=1}^{s} \operatorname{Ker} Q_{i}\right) \leq \sum_{i=0}^{s} \operatorname{codim} \Pi_{i} .
$$

Proof. It follows from (5.5) that

$$
\begin{gathered}
\bigcap_{i=1}^{s} \operatorname{Ker} Q_{i}=\left\{x=\left(x_{1}, x_{2}\right) \in R^{n}: A_{i} x_{1}=0 ; B_{i} x_{1}+A_{i}^{*} x_{2}=0, i=1, \ldots, s ;\right. \\
\left.x_{2} \in\left(\bigcap_{i=1}^{s} \operatorname{Ker} A_{i}^{*}\right)^{\perp}\right\} \oplus\{0\} \times \bigcap_{i=1}^{s} \operatorname{Ker} A_{i}^{*} .
\end{gathered}
$$

Here $x_{1} \in R^{n_{1}}$ are the first $n_{1}$ coordinates of the vector $x$ and $x_{2} \in R^{n_{2}}$ its last $n_{2}$ coordinates. From this and (5.6), we obtain

$$
\begin{gathered}
\operatorname{dim}\left(\bigcap_{i=1}^{s} \operatorname{Ker} Q_{i}\right) \geq \operatorname{dim}\left(\bigcap_{i=1}^{s} \operatorname{Ker} A_{i}^{*}\right) \\
+\operatorname{dim}\left\{x_{1} \in \Pi: \exists x_{2} \in R^{n_{2}}, B_{i} x_{1}+A_{i}^{*} x_{2}=0, i=1, \ldots, s\right\} .
\end{gathered}
$$

Let us consider the system of equations

$$
-A_{i}^{*} x_{2}=B_{i} x_{1}, i=1, \ldots, s,
$$

with respect to $x_{2}$. From the Fredholm theorem it follows [22, chapter 10, $\left.\S 85\right]$ that the system has a solution for any vector $x_{1} \in \Pi$ such that

$$
\sum_{i=1}^{s}\left\langle B_{i} \xi_{i}, x_{1}\right\rangle=0 \forall \tilde{\xi}=\left(\xi_{1}, \ldots, \xi_{s}\right): \xi_{i} \in R^{n_{1}}, \sum_{i=1}^{s} A_{i} \xi_{i}=0
$$

But by (5.6) and (5.7), it is enough to verify the theorem only for $\tilde{\xi}$ with $\xi_{i} \in \Pi_{i}^{\perp}$. Therefore those equations can be replaced by the following ones:

$$
\sum_{i=1}^{s}\left\langle B_{i} \xi_{i}, x_{1}\right\rangle=0 \forall \tilde{\xi}: \xi_{i} \in \Pi_{i}^{\perp}, i=1, \ldots, s ; \sum_{i=1}^{s} A_{i} \xi_{i}=0 .
$$

We shall consider (5.11) as a linear system with respect to $x_{1} \in R^{n_{1}}$ in order to determine the subspace $R$ that contains any vector $x_{1}$ for which (5.10) has a solution.

Let us count the number of equations in (5.11). It is easy to see that this number is equal to $\chi(s)$, where

$$
\chi(i)=\operatorname{dim}\left\{\tilde{\xi}=\left(\xi_{1}, \ldots, \xi_{s}\right): \xi_{l} \in \Pi_{l}^{\perp}, l=1, \ldots, i ; \sum_{l=1}^{i} A_{l} \xi_{l}=0\right\}, i=1, \ldots, s .
$$

We shall calculate $\chi(s)$. Let us show that

$$
\chi(s)=\sum_{i=1}^{s} \operatorname{codim}\left(\operatorname{Ker} A_{i}\right)-\operatorname{codim}\left(\bigcap_{i=1}^{s} \operatorname{Ker} A_{i}^{*}\right) .
$$


Indeed, we have

$$
\begin{gathered}
\chi(s)=\operatorname{dim}\left\{\operatorname{Im} A_{s} \cap \sum_{i=1}^{s-1} \operatorname{Im} A_{i}\right\}+\chi(s-1) \\
\stackrel{(1)}{=} \operatorname{dim}\left(\operatorname{Im} A_{s}\right)-\operatorname{dim}\left(\sum_{i=1}^{s} \operatorname{Im} A_{i}\right)+\operatorname{dim}\left(\sum_{i=1}^{s-1} \operatorname{Im} A_{i}\right)+\chi(s-1) \\
\stackrel{(2)}{=} \operatorname{dim}\left(\operatorname{Im} A_{s}\right)-\operatorname{dim}\left(\sum_{i=1}^{s} \operatorname{Im} A_{i}\right)+\operatorname{dim}\left(\sum_{i=1}^{s-1} \operatorname{Im} A_{i}\right) \\
+\operatorname{dim}\left(\operatorname{Im} A_{s-1}\right)+\operatorname{dim}\left(\sum_{i=1}^{s-2} \operatorname{Im} A_{i}\right)-\operatorname{dim}\left(\sum_{i=1}^{s-1} \operatorname{Im} A_{i}\right)+\chi(s-2) \\
=\ldots=\sum_{i=1}^{s} \operatorname{dim}\left(\operatorname{Im} A_{i}\right)-\operatorname{dim}\left(\sum_{i=1}^{s} \operatorname{Im} A_{i}\right)+\chi(1) \\
\stackrel{(3)}{=} \sum_{i=1}^{s} \operatorname{dim}\left(\operatorname{Im} A_{i}\right)-\operatorname{codim}\left(\bigcap_{i=1}^{s} \operatorname{Ker} A_{i}^{*}\right) .
\end{gathered}
$$

That proves (5.12). Here the equalities $\stackrel{(1)}{=}$ and $\stackrel{(2)}{=}$ follow from the formula

$$
\operatorname{dim}\left(R_{1} \cap R_{2}\right)=\operatorname{dim} R_{1}+\operatorname{dim} R_{2}-\operatorname{dim}\left(R_{1}+R_{2}\right),
$$

which is true for any two subspaces $R_{1}$ and $R_{2}$. The equality $\stackrel{(3)}{=}$ is a consequence of the fact that $\chi(1)=0$ and the known formulas

$$
R_{1}+R_{2}=\left(R_{1}^{\perp} \cap R_{2}^{\perp}\right)^{\perp} ;\left(\operatorname{Im} A_{i}\right)=\operatorname{Ker} A_{i}^{*} ; \operatorname{dim}\left(\operatorname{Im} A_{i}\right)=\operatorname{codim}\left(\operatorname{Ker} A_{i}\right) .
$$

The equations in (5.11) are not all independent. By (5.6) and (5.8), some of them hold for an arbitrary $x_{1} \in \Pi$. The number of such equations is not less than $\operatorname{dim} \Pi_{0}-\operatorname{dim} \Pi$.

Consequently, by (5.12), the number of the independent equations which define the subspace $R$ does not exceed the number

$$
\chi=\sum_{i=1}^{s} \operatorname{codim}\left(\operatorname{Ker} A_{i}\right)+\operatorname{dim} \Pi-\operatorname{codim}\left(\bigcap_{i=1}^{s} \operatorname{Ker} A_{i}^{*}\right)-\operatorname{dim} \Pi_{0} .
$$

Therefore, from (5.9), we get

$$
\begin{gathered}
\operatorname{dim}\left(\bigcap_{i=1}^{s} \operatorname{Ker} Q_{i}\right) \geq \operatorname{dim}\left(\bigcap_{i=1}^{s} \operatorname{Ker} A_{i}^{*}\right)+\operatorname{dim} \Pi-\chi \\
=n_{2}-\sum_{i=1}^{s} \operatorname{codim}\left(\operatorname{Ker} A_{i}\right)+\operatorname{dim} \Pi_{0} \geq n-\sum_{i=0}^{s} \operatorname{codim} \Pi_{i} .
\end{gathered}
$$

That proves the required inequality.

Here the last inequality holds because, by (5.6), $\operatorname{codim} \Pi_{i} \geq \operatorname{codim}\left(\operatorname{Ker} A_{i}\right)$, $i=1, \ldots, s$. The lemma is established. 
4. Let us prove Theorem 5.1 by contradiction. Suppose that $F$ is not 2-normal at the point $x_{0}$.

By Lemma 5.1, there exist a number $s \leq d$ and linearly independent vectors $y_{1}, \ldots, y_{s}$ satisfying (5.2), (5.3). Then without loss of generality we will consider $y_{i}=(0, \ldots, 1,0, \ldots, 0)$ with unity in the $i$ th place. Let $Q_{i}$ denote the restriction of the bilinear form $y_{i} \frac{\partial^{2} F}{\partial x^{2}}\left(x_{0}\right)=\frac{\partial^{2} F_{i}}{\partial x^{2}}\left(x_{0}\right)$ to $\operatorname{Ker} F^{\prime}\left(x_{0}\right)$ and $q_{i}$ the corresponding quadratic form, $i=1, \ldots, s$. On account of the first $s$ inequalities of (5.3), there exist subspaces $\tilde{\Pi}_{i} \subseteq \operatorname{Ker} F^{\prime}\left(x_{0}\right)$ such that the codimension of $\tilde{\Pi}_{i}$ in $X$ does not exceed $d$ and $q_{i}(x) \geq 0 \forall x \in \tilde{\Pi}_{i}, i=1, \ldots, s$. In the same way, from the last inequality of (5.3) we see that there exists a subspace $\tilde{\Pi}_{0} \subseteq \operatorname{Ker} F^{\prime}\left(x_{0}\right)$ such that the codimension of $\tilde{\Pi}_{0}$ does not exceed $d$ and $\sum_{i=1}^{s} q_{i}(x) \leq 0 \forall x \in \tilde{\Pi}_{0}$. Let us set $\tilde{\Pi}=\bigcap_{i=0}^{s} \tilde{\Pi}_{i}$. Then it is clear that every form $q_{i}$ vanishes on $\tilde{\Pi}$, and the codimension of $\operatorname{Ker} F^{\prime}\left(x_{0}\right)$ does not exceed $d(s+1)$. It follows that the corresponding bilinear forms $Q_{i}, i=1, \ldots, s$, vanish on $\tilde{\Pi}$, and therefore $\tilde{Q}[\bar{x}, \hat{x}]=0 \forall \bar{x}, \hat{x} \in \tilde{\Pi}$. Here $\tilde{Q}: \operatorname{Ker} F^{\prime}\left(x_{0}\right) \times \operatorname{Ker} F^{\prime}\left(x_{0}\right) \rightarrow R^{s}$ is a bilinear mapping with coordinates $Q_{1}, \ldots$, $Q_{s}$. It follows easily that $\operatorname{dim}(N \tilde{\Pi}) \leq d$ and hence the codimension of $\operatorname{Ker} N$ is finite. Here $N$ is the linear operator generated by the bilinear mapping $\tilde{Q}$. Cosequently, while proving the theorem, one can assume without loss of generality that the space $X$ itself is finite-dimensional and the space $\operatorname{Ker} F^{\prime}\left(x_{0}\right)$ is equal to the $n$-dimensional arithmetic space $R^{n}$ for a positive integer $n$.

Let $n_{1}$ denote the codimension of $\tilde{\Pi}$ in $R^{n}$, and set $n_{2}=n-n_{1}$. Every form $q_{i}$ vanishes on $\tilde{\Pi}$. Hence, we use a linear nondegenerate transformation of $R^{n}$ such that after it every matrice $Q_{i}$ defining the bilinear form $Q_{i}$ (they are denoted by the same symbol) will have the block form (5.5). Then the quadratic form $q_{i}$ can be represented in the following way:

$$
q_{i}(x)=B_{i}\left[x_{1}\right]^{2}+2\left\langle A_{i} x_{1}, x_{2}\right\rangle \forall x=\left(x_{1}, x_{2}\right) \in R^{n} .
$$

Here $x_{1}$ are the first $n_{1}$ coordinates of the vector $x, x_{2}$ the other coordinates, and $R^{n}=R^{n_{1}} \times R^{n_{2}}$

Let us fix $i$ and consider the quadratic form $q\left(x_{1}\right)=B_{i}\left[x_{1}\right]^{2}$ on the space Ker $A_{i}$. We shall prove that its index does not exceed the number $\rho=d-\operatorname{codim}\left(\operatorname{Ker} A_{i}\right)$. Indeed, assume the opposite. Then there exists a subspace $Z$ in Ker $A_{i}$ such that $q$ is negative definite on it and $\operatorname{dim} Z=\rho+1$. Set

$$
\begin{gathered}
\kappa_{1}=\max \left\{q\left(x_{1}\right): x_{1} \in Z,\left|x_{1}\right|=1\right\} ; \\
\kappa_{2}=\min \left\{\left|A_{i} x_{1}\right|^{2}: x_{1} \in\left(\operatorname{Ker} A_{i}\right)^{\perp},\left|x_{1}\right|=1\right\} .
\end{gathered}
$$

It is evident that $\kappa_{1}<0, \kappa_{2}>0$. We will choose an arbitrary $\delta>0$ and consider the quadratic form $q_{i}$ on the subspace

$$
P_{\delta}=Z \times\{0\} \oplus\left\{\left(x_{1}, x_{2}\right): x_{1} \in\left(\operatorname{Ker} A_{i}\right)^{\perp}, x_{2}=-\delta A_{i} x_{1}\right\} .
$$

We shall show that if $\delta$ is sufficiently large, then $q_{i}$ is negative definite on $P_{\delta}$. Indeed, $x=\left(x_{1}, x_{2}\right) \in P_{\delta} \Rightarrow x_{1}=\bar{x}_{1}+\hat{x}_{1}$. Here $\bar{x}_{1} \in Z, \hat{x}_{1} \in\left(\operatorname{Ker} A_{i}\right)^{\perp}$, and $x_{2}=-\delta A_{i} \hat{x}_{1}$. According to (5.13), we get

$$
\begin{gathered}
q_{i}(x)=B_{i}\left[x_{1}\right]^{2}-2 \delta\left|A_{i} \hat{x}_{1}\right|^{2} \\
\leq \kappa_{1}\left|\bar{x}_{1}\right|^{2}+\left(\kappa_{1}-2 \delta \kappa_{2}\right)\left|\hat{x}_{1}\right|^{2}+\text { const }\left|\bar{x}_{1}\right|\left|\hat{x}_{1}\right|
\end{gathered}
$$

(const is a positive constant). But the quadratic polynomial in the variables $\left|\bar{x}_{1}\right|$ and $\left|\hat{x}_{1}\right|$ taking up the right part of that inequality turns out to be negative definite 
for large $\delta>0$ because $\kappa_{1}<0, \kappa_{2}>0$. Hence, we choose a large $\delta>0$ such that the form $q_{i}$ is negative on the subspace $P_{\delta}$, whose dimension is obviously equal to $(d+1)$ by definition. Therefore, ind $q_{i} \geq d+1$, which is impossible by (5.3). This contradiction proves that the index of the form $q$ on $\operatorname{Ker} A_{i}$ does not exceed $\rho$. That implies the existence of a subspace $\Pi_{i} \subseteq R^{n_{1}}$ such that for $i \in\{1, \ldots, s\}$

$$
\Pi_{i} \subseteq \operatorname{Ker} A_{i} ; \operatorname{codim} \Pi_{i} \leq d ; q_{i}(x) \geq 0 \forall x \in \Pi_{i} \times R^{n_{2}} .
$$

(Now codim is codimension in $R^{n_{1}}$ ). In a similar way one can prove the existence of a subspace $\Pi_{0} \subseteq R^{n_{1}}$ such that

$$
\Pi_{0} \subseteq \operatorname{Ker}\left(\sum_{i=1}^{s} A_{i}\right) ; \operatorname{codim} \Pi_{0} \leq d ; \sum_{i=1}^{s} q_{i}(x) \leq 0 \forall x \in \Pi_{0} \times R^{n_{2}} .
$$

Set $\Pi=\bigcap_{i=0}^{s} \Pi_{i}$. Then the form $B_{i}\left[x_{1}\right]^{2}$ is nonnegative on $\Pi_{i}, i=1, \ldots, s$, and vanishes on $\Pi$. From this and (5.13)-(5.15) we see that the conditions (5.7) and (5.8) are satisfied. Hence, the matrices $Q_{i}, i=1, \ldots, s$, satisfy all the assumptions of Lemma 5.2. Consequently,

$$
\operatorname{codim}\left(\bigcap_{i=1}^{s} \operatorname{Ker} Q_{i}\right) \leq d(s+1),
$$

for, as we proved earlier, codim $\Pi_{i} \leq d \forall i$. But this contradicts the assumption (5.4) of the theorem. Hence, the mapping $F$ is 2-normal at the point $x_{0}$. The theorem is established.

5. Definition. The mapping $F$ is called 2-normal if it is 2-normal with respect to an arbitrary cone $C$ at every point $x \in X$.

Let $X=R^{n}$ and $F$ be a three times continuously differentiable mapping. We shall show that if the dimension $n$ is significantly greater than the number of constraints $k$ (see (5.16) below), then a 2-normal mapping $F$ is generic.

Consider the linear topological space $C_{s}^{3}\left(R^{n}, R^{k}\right)$ of three times continuously differentiable mappings $F: R^{n} \rightarrow R^{k}$ provided with the Whitney topology [19, chapter $2, \S 1]$. Recall that the basis of the Whitney topology consists of sets $W$ of the following type:

$$
\begin{aligned}
W & =W\left(\left\{K_{i}\right\},\left\{U_{i}\right\},\left\{\varepsilon_{i}\right\}\right) \\
& =\left\{g \in C_{s}^{3}\left(R^{n}, R^{k}\right):\left|D^{j} F(x)-D^{j} g(x)\right|<\varepsilon_{i} \forall x \in K_{i}, j=0,1,2,3\right\} .
\end{aligned}
$$

Here $K_{i}$ are compact sets contained in $U_{i},\left\{U_{i}\right\}$ is a locally finite open covering of $R^{n}$ and $\left\{\varepsilon_{i}\right\}$ a family of positive numbers.

Theorem 5.2. Let

$$
n>2(k-2) ; \quad(n-k-1)(n-k)>2(k-1) .
$$

Then the set of 2-normal mappings is residual (i.e. it contains the intersection of countable everywhere dense open sets) and it is therefore everywhere dense in $C_{s}^{3}\left(R^{n}, R^{k}\right)$.

Proof. We will use the techniques and terminology of differential topology (see [19, chapters 2,3]). We first remark that $C_{s}^{3}\left(R^{n}, R^{k}\right)$ is a Baire space [19, theorem 4.4]. Therefore it is sufficient to prove that the set of 2-normal mappings is residual.

Let $J^{2}(n, k)$ denote the space of 2 -jets from $R^{n}$ to $R^{k}$. Elements of $J^{2}(n, k)$ may be identified with collections $\gamma=(x, y, A, B)$. Here $x \in R^{n}, y \in R^{k}, A$ is an $n \times k$ matrix, $B=\left(B_{1}, \ldots, B_{k}\right)$, and $B_{i}$ are $n \times n$ symmetric matrices. 
Consider an arbitrary positive integer $r \leq k-2$, and let $T_{r}$ denote the set of those elements $\gamma \in J^{2}(n, k)$ for which $\operatorname{rank} A=r$. Furthermore, for $l \in\{0,1,2\}$ every $T_{k-1, l}$ denotes a subset of $\gamma \in J^{2}(n, k)$ such that

$\operatorname{rank} A=k-1, \exists z \in R^{k}: A z=0, z \neq 0, \operatorname{codim}(\operatorname{Ker} B z)=\operatorname{rank} B z=l$.

Here $B z=\sum_{i=1}^{k} B_{i} z^{i}$.

As it is known [19], each set $T_{r}, r=0, \ldots, k-2$, is a smooth submanifold of $J^{2}(n, k)$ with codimension equal to $(n-r)(k-r)$. Therefore

$$
\operatorname{codim} T_{r} \geq 2(n-k+2), \quad r=0, \ldots, k-2 .
$$

We will prove that $T_{k-1, l}, l=0,1,2$, are also smooth submanifolds of $J^{2}(n, k)$ and calculate their codimensions. Let $\mathcal{A}$ denote the set of $n \times k$ matrices of rank $k-1$. It is a smooth submanifold in the space of $n \times k$ matrices, and $\operatorname{codim} \mathcal{A}=n-k+1$. Furthermore, without loss of generality, we suppose that the vector $z$ of (5.17) is an element of the projective space $R P^{k-1}$. Consequently, taking into account that $\operatorname{rank} A=k-1$ in (5.17), we conclude that a vector $z \in R P^{k-1}$ with $A z=0$ is unique and smoothly depends on $A$ as on a point of the smooth submanifold $\mathcal{A}$. Let us denote that vector $z$ by $z(A)$.

Thus, $\gamma \in T_{k-1, l} \Leftrightarrow A \in \mathcal{A}, B z(A) \in \mathcal{B}_{l}$. Here $\mathcal{B}_{l}$ is the set of $n \times n$ symmetric matrices of rank $l$. But in the space of $n \times n$ symmetric matrices $\mathcal{B}_{l}$ is a smooth submanifold and $\operatorname{codim} \mathcal{B}_{l}=\frac{1}{2}(n-l)(n-l+1)$ (this follows, for example, from the reasoning of [20, p.24]). Employing the implicit function theorem to find $B$ such that $B z(A) \in \mathcal{B}_{l}$ and using the smoothness of the function $z(\cdot)$, we deduce that the set $T_{k-1, l}$ is a smooth submanifold of $J^{2}(n, k)$ and $\operatorname{codim} T_{k-1, l}=\operatorname{codim} \mathcal{A}+$ $\operatorname{codim} \mathcal{B}_{l} \Rightarrow$

$$
\operatorname{codim} T_{k-1, l} \geq(n-k+1)+\frac{1}{2}(n-k)(n-k-1) \forall l .
$$

Let $\tilde{T}$ denote the set of the submanifolds $T_{r}, r=0, \ldots, k-2$, and $T_{k-1, l}$, $l=0,1, \ldots, k+1$. Therefore we proved that every set $T \in \tilde{T}$ is a smooth submanifold of $J^{2}(n, k)$. Moreover, from (5.16), (5.18), (5.19) we see that the codimension of each submanifold is greater than $n$. From this it follows immedeately that if for a mapping $F \in C_{s}^{3}\left(R^{n}, R^{k}\right)$ its jet extension $j^{2} F$ is transversal to a manifold $T \in \tilde{T}$, then its 2-jet $j^{2} F(x)$ at an arbitrary point $x \in R^{n}$ does not belong to that manifold. But $\tilde{T}$ consists of a finite number of submanifolds. Thus, according to the jet theorem on transversality [19], we see that the set of mappings $F \in C_{s}^{3}\left(R^{n}, R^{k}\right)$ whose jet extensions are transversal to each manifold $T \in \tilde{T}$ is residual. Consequently, the set of the mappings $F$ whose 2-jet $j^{2} F(x)$ at any point does not belong to any set $T \in \tilde{T}$ is residual and everywhere dense in $C_{s}^{3}\left(R^{n}, R^{k}\right)$. From the definition of the manifolds $T_{r}$ and $T_{k-1, l}$ and Theorem 5.1 it follows that the mapping $F$ is 2-normal at a point $x$ with respect to any cone $C$ if the 2 -jet $j^{2} F(x)$ of the mapping $F$ at the point $x$ does not belong to any set $T \in \tilde{T}$. The theorem is proved.

Theorem 5.2 shows the main distinction of the condition of 2-normality from the conditions of normality (the Lyusternik condition) and of 2-regularity. For $F$ 
being 2-normal - as opposed to the above-mentioned and analogous nondegeneracy conditions - means being generic in the space of functions $C_{s}^{3}\left(R^{n}, R^{k}\right)$.

Finally, the author would like to thank the anonymous referee for helpful suggestions.

\section{REFERENCES}

1. G.A. Bliss, Lectures on the Calculus of Variations, Univ. Chicago Press, Chicago (1946). MR 8:212e

2. R. Henrion, "La Théorie de la Seconde Variation et ses Applications en Comande Optimale", Acad. Roy. Belg. Cl. Sci. Mem. Coll. in 8 (2), 41 No 7, (1975). MR 55:5267

3. A.D. Ioffe and V.M. Tikhomirov, Theory of Extremal Problems, North - Holland, Amsterdam (1979). MR 80d:49001b

4. N. Bourbaki, Topologie Generale, Chap.1-2, Hermann, Paris (1961). MR 25:4480

5. A.V. Arutyunov, "Perturbations of Extremal Problems with Constraints and Necessary Conditions of Optimality", Itogi Nauki i Tekhniki. Ser. Matem. Analiz, 27, 147-235, (1989); English transl., J. Soviet Math. 54 (1991), 1342-1400. MR 91a:49016

6. V.M. Alekseev, V.M.Tikhomirov and S.V.Fomin, Optimal Control, Consultants Bureau, New York (1987). MR 89e:49002

7. B.N. Pshenichnii, Convex Analysis and Extremal Problems (in Russian), Nauka, Moscow (1980). MR 81k:90080

8. A.A.Agrachev, "The Topology of Quadratic and Smooth Mappings", Itogi Nauki i Tekhniki. Ser. Algebra. Topologiya. Geometriya, 26, 85-124, (1988); English transl., J. Soviet Math. 49 (1990), 990-1013. MR 90b:58032

9. A.V. Arutyunov, "Necessary Extremal Conditions for Abnormal Problem with Constraints of Equality Type", Usp. Mat. Nauk, 45, No 5, 181-182 (1990); English transl., Russian Math. Surveys 45, No. 5 (1990), 223-224. MR 92b:49043

10. L.L. Dines, "On the Mapping of $n$ Quadratic Forms", Bull. Amer. Math. Soc., Vol. 48, 467471 (1942). MR 3:261b

11. A.V. Arutyunov, "On Necessary Conditions of Optimality Problem with Phase Constraints", Dokl. Akad. Nauk SSSR, 280, No 5, 1033-1037 (1985); English transl., Soviet Math. Dokl. 280 (1985), 174-177. MR 86f:49073

12. E.R. Avakov, A.A. Agrachev, A.V. Arutyunov, "The Level Set of a Smooth Mapping in a Neighbourhood of a Singular Point and the Zeros of a Quadratic Mapping", Mat. Sb., 182, No 8, 1091-1104 (1991); English transl., Math. USSR Sb. 73 (1992), 455-466. MR 92k:58028

13. A.V. Arutyunov and N.T. Tynyanskii, "On Necessary Conditions for a Local Minimum in Optimal Control Theory", Dokl. Akad. Nauk SSSR, 275, 268-272, (1984); English transl., Soviet Math. Dokl. 29 (1984), 176-179. MR 85m:49034

14. A.A. Agrachev "Another Condition for a Conditional Extremum", Usp. Mat. Nauk, 44, No 5, 153-154 (1989); English transl., Russian Math. Surveys 44, No. 5 (1989), 189-190. MR 91c:90095

15. E.S. Levitin, A.A.Milyutin and N.P.Osmolovskii, "Higher Order Conditions for Local Minimum for Problems with Constraints", Usp. Mat. Nauk, 33, No 6, 85-148, (1978); English transl., Russian Math. Surveys 33, no. 6 (1978), 97-168. MR 80f:49001

16. A.A.Milyutin, "On Quadratic Conditions for an Extremum for Smooth Problems with Finitedimensional Image", in: Methods of the Theory of Extremal Problems in Economics [in Russian], Nauka, Moscow, 137-177 (1981). MR 84g:90039

17. E.R. Avakov, "Conditions for an Extremum for Smooth Problems with Constraints of Equality Type", Zh. Vychisl. Mat. Mat. Fiz., 25, No 5, 680-693 (1985); English transl., USSR Comput. Math. and Math. Phys. 25 (1985), No. 3, 114-121. MR 86j:49056

18. R. Tyrrell Rockafellar, Convex Analysis, Princeton Univ. Press, (1970). MR 43:445

19. Morris W. Hirsch, Differential topology, Springer-Verlag, New York, Heidelberg, Berlin, (1976). MR 56:6669

20. V.I. Arnold, A.N. Varchenko, S.M. Gusein-Zade, Singularities of Differentiable Mappings, [in Russian] Nauka, Moscow (1982); English transl., Birkhäuser, 1985. MR 84m:58016; MR 86f: 58018 
21. A.V. Finkelshtein, "Application of the Ekeland theorem for the derivation of necessary and sufficient conditions of optimality for sequences" in: Optimizatsiia, 31(48) [in Russian], Novosibirsk, 33-47 (1983). MR 86c:49040

22. V.V.Voevodin, Linear Algebra, Nauka, Moscow [in Russian] (1974). MR 50:9894

23. A.R. Avakov, Necessary Extremum Conditions for Smooth Abnormal Problem with Equality and Inequality-type Constraints, Mat. Zametki, 45, No 6, 3-11, (1989); English transl., Math. Notes 45 (1989), 431-437. MR 90k:49024

Department of Differentional Equations and Functional Analysis, Peoples FriendShip University of Russia, Moscow 117198, Mikluka-Maklai, 6, Russia

E-mail address: arutunov@sa640.cs.msu.su 